\title{
REGULARIZED THETA LIFTS FOR ORTHOGONAL GROUPS OVER TOTALLY REAL FIELDS
}

\author{
JAN HENDRIK BRUINIER
}

\begin{abstract}
We define a regularized theta lift from $\mathrm{SL}_{2}$ to orthogonal groups over totally real fields. It takes harmonic 'Whittaker forms' to automorphic Green functions and weakly holomorphic Whittaker forms to meromorphic modular forms on orthogonal groups with zeros and poles supported on special divisors, generalizing Borcherds' work on automorphic products. To prove our results we use the spectral expansion of the lift and study its relationship with the cohomological theta lift of Kudla and Millson.
\end{abstract}

\section{INTRODUCTION}

The theory of dual reductive pairs and theta liftings provides an important tool for the construction of automorphic forms and for understanding the relationship between automorphic forms on different groups. A new aspect was added to the theory by the celebrated discovery of Harvey-Moore and Borcherds that divergent theta integrals can often be regularized [HM], Bo1]. One can define a regularized theta lift of (vector valued) weakly holomorphic modular forms for $\mathrm{SL}_{2}(\mathbb{Z})$ to meromorphic modular forms on orthogonal groups associated to rational quadratic spaces of signature $(n, 2)$. These lifts have their zeros and poles on special divisors (also referred to as Heegner divisors or rational quadratic divisors). Their Fourier expansions are given by infinite products, so called Borcherds products. They have found various applications, for instance in the theory of generalized Kac-Moody algebras, in the study of moduli problems, and in the geometry and arithmetic of Shimura varieties, see e.g. [AF, [Bo2, [Bo3], [GN], [Ku3], [BBK], BY1].

Since the work of Borcherds, there has been the question whether the regularized theta lift can be generalized to dual reductive pairs other than $\left(\mathrm{SL}_{2}, \mathrm{O}(V)\right)$, where $V$ is a quadratic space over $\mathbb{Q}$ (see [Bo1], Problem 16.4). For instance, one would like to define it for quadratic spaces over totally real number fields as well. There are two serious problems that arise. First, the special cycles on which the lift should have its singularities are not divisors in general, so one cannot expect that they are related to a single meromorphic function. Second, the straightforward generalization of weakly holomorphic elliptic modular forms, the "input" for the lift, would be meromorphic Hilbert modular forms whose poles are supported at the Baily-Borel boundary. However, by the Koecher principle, there are no non-trivial modular forms of this type.

Date: March 1, 2022.

2000 Mathematics Subject Classification. 11F55, 11G18, $14 \mathrm{G} 35$.

The author is partially supported by DFG grant BR-2163/2-1. 
In the present paper, we propose a solution for the second problem. We introduce the notion of a weakly holomorphic Whittaker form and consider a regularized theta lift of such functions. It leads to meromorphic modular forms with singularities along special divisors, generalizing Borcherds' construction of automorphic products [Bo1]. More generally, we study harmonic Whittaker forms and their regularized theta lifts. They give rise to Green functions in the sense of Arakelov geometry (cf. [SABK], [BKK]).

We now describe the content of this paper in more detail. Let $F$ be a totally real number field of degree $d$ and discriminant $D$. We write $\hat{F}$ for the ring of finite adeles and $\mathcal{O}_{F}$ for the ring of integers of $F$. Let $(V, Q)$ be a quadratic space over $F$ of dimension $\ell=n+2$ and assume that the signature of $V$ at the archimedian places of $F$ is equal to

$$
((n, 2),(n+2,0), \ldots,(n+2,0)) .
$$

We consider the algebraic group $H=\operatorname{Res}_{F / \mathbb{Q}} \operatorname{GSpin}(V)$ over $\mathbb{Q}$ given by Weil restriction of scalars. Our hypothesis on the signature guarantees that the symmetric space $\mathbb{D}$ associated to $H(\mathbb{R})$ carries an invariant hermitean structure and that there exist special divisors in the sense of $\mathrm{Ku} 1$.

Let $L \subset V$ be an even $\mathcal{O}_{F}$-lattice. To simplify the exposition we assume throughout this introduction that $L$ be unimodular. This implies in particular that $n$ is even. The case of arbitrary even lattices of possibly odd rank is treated in the body of the paper. For a compact open subgroup $K \subset H(\hat{\mathbb{Q}})$ stabilizing $L$ we consider the Shimura variety

$$
X_{K}=H(\mathbb{Q}) \backslash(\mathbb{D} \times H(\hat{\mathbb{Q}})) / K .
$$

It is a quasi-projective variety of dimension $n$ defined over $F$, see [Ku1]. It is projective if and only if $V$ is anisotropic over $F$. By our assumption on the signature of $V$ this is always the case if $d>1$.

Associated to $L$ there is a Siegel theta function $\Theta_{S}(\tau, z, h)$, where $\tau=u+i v \in \mathbb{H}^{d}$, $z \in \mathbb{D}$, and $h \in H(\hat{\mathbb{Q}})$, see Section 3. In the variable $\tau$ it transforms as a nonholomorphic Hilbert modular form of weight $\left(\frac{n-2}{2}, \frac{n+2}{2}, \ldots, \frac{n+2}{2}\right)$ for the group $\Gamma=\mathrm{SL}_{2}\left(\mathcal{O}_{F}\right)$. In the variable $(z, h)$ it is $H(\mathbb{Q})$-invariant. For a Hilbert modular form $f$ which is holomorphic in $\tau_{1}$ and antiholomorphic in $\tau_{2}, \ldots, \tau_{d}$ of weight $k=:\left(\frac{2-n}{2}, \frac{n+2}{2}, \ldots, \frac{n+2}{2}\right)$, we would like to consider the theta integral

$$
\phi(z, h, f)=\frac{1}{\sqrt{D}} \int_{\Gamma \backslash \mathbb{H}^{d}} f(\tau) \Theta_{S}(\tau, z, h)\left(v_{2} \cdots v_{d}\right)^{\ell / 2} d \mu(\tau) .
$$

However, if $n \geq 2$ then there are no non-constant Hilbert modular forms of this type with moderate growth at the cusps. In view of Borcherds' work on regularized theta lifts for $F=\mathbb{Q}$ one could try to look at the integral for Hilbert modular forms with singularities at the cusps. But the Koecher principle implies that there are no non-constant forms of this type when $d>1$. Now one could further relax the assumptions on $f$ and allow singularities on some divisor in addition to the cusps. However, then the lift tends to behave poorly under the invariant differential operators for $H(\mathbb{R})$.

Instead, we use a different approach here. To motivate it, we briefly revisit the case where $F=\mathbb{Q}$. For simplicity we assume in this paragraph that $n>2$. Any weakly 
holomorphic modular form of weight $k=\frac{2-n}{2}$ for $\Gamma=\mathrm{SL}_{2}(\mathbb{Z})$ can be constructed as a Poincaré series from a Whittaker form. For $s \in \mathbb{C}$ and $v \in \mathbb{R} \backslash\{0\}$ we let

$$
\mathcal{M}_{s}(v)=|v|^{-k / 2} M_{\operatorname{sgn}(v) k / 2, s / 2}(|v|) \cdot e^{-v / 2},
$$

where $M_{\nu, \mu}$ denotes the usual Whittaker function, and for any positive integer $m$ we put

$$
f_{m}(\tau, s)=\Gamma(s+1)^{-1} \mathcal{M}_{s}(-4 \pi m v) e(-m \bar{\tau}),
$$

where $e(u):=e^{2 \pi i u}$. A harmonic Whittaker form $f$ of weight $k$ is a finite linear combination of the functions $f_{m}(\tau, 1-k$ ) with $m>0$ (see Remark 4.2 for a characterization by differential equations and growth conditions). Note that such a function is annihilated by the hyperbolic Laplacian in weight $k$ and has exponential growth as $v \rightarrow \infty$. There is a differential operator $\xi_{k}$ taking harmonic Whittaker forms of weight $k$ to cusp forms of weight $2-k$. It is defined by

$$
\xi_{k}(f)=\left.2 i \sum_{\gamma \in \Gamma_{\infty} \backslash \Gamma} v^{k} \overline{\frac{\partial}{\partial \bar{\tau}}(f)}\right|_{2-k} \gamma
$$

where $\Gamma_{\infty}=\left\{\left(\begin{array}{ll}1 & b \\ 0 & 1\end{array}\right) ; b \in \mathbb{Z}\right\}$ denotes the subgroup of translations of $\Gamma$. We call $f$ weakly holomorphic if $\xi_{k}(f)=0$. If $f$ is a harmonic Whittaker form of weight $k$, then the Poincaré series

$$
\eta(f)=\left.\sum_{\gamma \in \Gamma_{\infty} \backslash \Gamma} f\right|_{k} \gamma
$$

converges and defines a harmonic weak Maass form of weight $k$ for $\Gamma$ in the sense of $[\mathrm{BF}]$. The assignment $f \mapsto \eta(f)$ defines an isomorphism between the space of harmonic Whittaker forms and the space of harmonic weak Maass forms of weight $k$. Moreover, $\eta(f)$ is a weakly holomorphic modular form if and only if $f$ is a weakly holomorphic Whittaker form (see Proposition 4.6). Many properties of harmonic weak Maass forms and their relationship to weakly holomorphic modular forms and cusp forms can also be rephrased using Whittaker forms, see Section 4 for details.

If $f$ is a harmonic Whittaker form and $\eta(f)$ the corresponding weak Maass form, we can unfold the regularized theta integral

$$
\begin{aligned}
\Phi(z, h, \eta(f)) & =\int_{\Gamma \backslash \mathbb{H}} \eta(f)(\tau) \Theta_{S}(\tau, z, h) d \mu(\tau) \\
& =\int_{\Gamma_{\infty} \backslash \mathbb{H}} f(\tau) \Theta_{S}(\tau, z, h) d \mu(\tau) .
\end{aligned}
$$

Consequently, regularized theta lifts of weakly holomorphic elliptic modular forms can also be viewed as regularized theta lifts of weakly holomorphic Whittaker forms.

We now come back to the more general setup above for an arbitrary totally real field $F$ of degree $d$. The idea of the present paper is that Whittaker forms have a straightforward generalization to this situation (see Definitions 4.1 and 4.4). Since they are only invariant under translations there is no Koecher principle. If $d>1$, then Poincaré series analogous 
to (1.1) diverge wildly. Nevertheless, in view of (1.2) we consider for a Whittaker form $f$ of weight $k$ the theta integral

$$
\Phi(z, h, f)=\frac{1}{\sqrt{D}} \int_{\Gamma_{\infty} \backslash \mathbb{H}^{d}} f(\tau) \Theta_{S}(\tau, z, h)\left(v_{2} \cdots v_{d}\right)^{\ell / 2} d \mu(\tau),
$$

where $\Gamma_{\infty}=\left\{\left(\begin{array}{ll}1 & b \\ 0 & 1\end{array}\right) ; b \in \mathcal{O}_{F}\right\}$ denotes the subgroup of translations of $\Gamma=\mathrm{SL}_{2}\left(\mathcal{O}_{F}\right)$. Since Whittaker forms are exponentially increasing as $v_{1} \rightarrow \infty$, the integral has to be regularized.

In Section 5 we define the regularization and study its properties. It suffices to do this for the functions $f_{m}(\tau, 1-k)$ for $m$ totally positive, see (4.13). If $\Re(s)$ is sufficiently large, then the theta integral of $f_{m}(\tau, s)$ can be regularized by first integrating over $u$ and afterwards over $v$, see Definition 5.1. The resulting function $\Phi_{m}(z, h, s)$ is an eigenfunction of the invariant Laplacian on $\mathbb{D}$ with a singularity along the special divisor $Z(m)$ of discriminant $m$. By means of ideas of Oda and Tsuzuki [OT] we compute the spectral expansion of $\Phi_{m}(z, h, s)$ and employ it to derive a meromorphic continuation to the whole $s$-plane and a functional equation in $s$ (see Theorems 5.8 and 5.12). We define the regularized theta integral of $f_{m}(\tau, 1-k)$ as the constant term in the Laurent expansion of $\Phi_{m}(z, h, s)$ at $s=1-k$. So for any harmonic Whittaker form

$$
f=\sum_{m \gg 0} c(m) f_{m}(\tau, 1-k)
$$

of weight $k$ we obtain a regularized theta lift $\Phi(z, h, f)$.

Theorem 1.1. (See Theorem 5.14.) The regularized theta lift $\Phi(z, h, f)$ of $f$ is a logarithmic Green function in the sense of Arakelov geometry for the divisor

$$
Z(f)=\sum_{m \gg 0} c(m) Z(m) .
$$

In Section 6 we investigate the relationship of the regularized theta lift and the KudlaMillson lift (see e.g. [KM3]). Recall that Kudla and Millson constructed a theta function $\Theta_{K M}(\tau, z, h)$ which transforms in $\tau$ like a non-holomorphic Hilbert modular form of weight $\kappa=\left(\frac{n+2}{2}, \ldots, \frac{n+2}{2}\right)$ and which takes values in the closed differential forms of type $(1,1)$ on $X_{K}$. For a Hilbert cusp form $g$ of weight $\kappa$ we may consider the theta integral

$$
\Lambda(z, h, g)=\frac{1}{\sqrt{D}} \int_{\Gamma \backslash \mathbb{H}^{d}} \overline{g(\tau)} \Theta_{K M}(\tau, z, h) v^{\kappa} d \mu(\tau) .
$$

It gives rise to a map from Hilbert cusp forms to closed harmonic $(1,1)$-forms on $X_{K}$. Using the action of various differential operators on the Siegel and the Kudla-Millson theta kernels as in $[\mathrm{BF}$, we prove (see Theorem 6.4):

Theorem 1.2. Let $f$ be a harmonic Whittaker form of weight $k$ for $\Gamma$. Then

$$
d d^{c} \Phi(z, h, f)=\Lambda\left(z, h, \xi_{k}(f)\right)-B(f) \Omega .
$$

Here $B(f)$ is a constant which is explicitly given by the Fourier coefficients of a certain Hilbert Eisenstein series of weight $\kappa$ and $\Omega$ denotes the invariant Kähler form on $\mathbb{D}$. 
If $f$ is weakly holomorphic, then $\xi_{k}(f)=0$. Hence the first term on the right hand side vanishes and $\Phi(z, h, f)$ is essentially a pluriharmonic function. This can be used to prove the main result of the present paper (Theorem 6.8).

Theorem 1.3. Let $f$ be a weakly holomorphic Whittaker form of weight $k$ for $\Gamma$ as in (1.3). Assume that the coefficients $c(m)$ are integral. Then there exists a meromorphic modular form $\Psi(z, h, f)$ for $H(\mathbb{Q})$ of level $K$ with a multiplier system of finite order such that:

(i) The weight of $\Psi$ is $-B(f)$.

(ii) The divisor of $\Psi$ is equal to $Z(f)$.

(iii) The Petersson metric of $\Psi$ is given by

$$
-\log \|\Psi(z, h, f)\|_{P e t}^{2}=\Phi(z, h, f) .
$$

Up to the statement about the Fourier expansion of $\Psi$, this result is completely analogous to Theorem 13.3 of [Bo1] on the regularized theta lift of weakly holomorphic modular forms. When $d=1$, it is compatible via the map $\eta$ with Borcherds' result (and gives a new proof of it). Notice that when $d>1$, the variety $X_{K}$ is compact and there are no Fourier expansions.

The third assertion of Theorem 1.3 provides a regularized integral representation for $\log \|\Psi\|_{P e t}^{2}$. In a follow-up paper [BY2] this is used to compute CM values and integrals of $\log \|\Psi\|_{\text {Pet }}^{2}$, and more generally of the Green functions $\Phi(z, h, f)$, extending results of [Ku3], [Scho, [BY1], BK] to totally real fields. Such quantities can be interpreted as archimedian intersection pairings and therefore play an important role in arithmetic intersection theory.

Theorem 1.3 can be used (when $n>2$ ) to show that the generating series

$$
A(\tau)=-c_{1}\left(\mathcal{M}_{1}\right)+\sum_{m \gg 0} Z(m) q^{m}
$$

of the special divisors $Z(m)$ is a Hilbert modular form of weight $\kappa$ with values in the first Chow group of $X_{K}$ (see Theorem [7.1). Here $c_{1}\left(\mathcal{M}_{1}\right)$ denotes the Chern class of the line bundle of modular forms of weight 1. Our proof is a variant of the proof that Borcherds gave for $F=\mathbb{Q}$, see [Bo2]. This result was also proved in [YZZ] using the modularity result of Kudla-Millson [KM3] for the cohomology classes of special divisors.

In Section 8 we present some examples illustrating Theorem 1.3. In particular, we consider Shimura curves over totally real fields and Shimura varieties associated to orthogonal groups of even unimodular lattices over real quadratic fields.

I thank A. Deitmar for his help with Lemma 5.11 and G. Nebe for her help with Section 8.2. Moreover, I thank E. Freitag, J. Funke, K. Ono and T. Yang for many useful conversations and comments related to this paper.

\section{QuAdratic SPACES AND Shimura VARIETiES}

Throughout we use the setup of [Ku1, Section 1 . Let $F$ be a totally real number field of degree $d$ over $\mathbb{Q}$. We write $\mathcal{O}_{F}$ for the ring of integers in $F$, and write $\partial=\partial_{F}$ for the different ideal of $F$. The discriminant of $F$ is denoted by $D=\mathrm{N}\left(\partial_{F}\right)=\# \mathcal{O}_{F} / \partial_{F}$. Let 
$\sigma_{1}, \ldots, \sigma_{d}$ be the different embeddings of $F$ into $\mathbb{R}$. We write $\mathbb{A}_{F}$ for the ring of adeles of $F$ and $\hat{F}$ for the subring of finite adeles. Moreover, we put $\mathbb{A}=\mathbb{A}_{\mathbb{Q}}$.

Let $(V, Q)$ be a non-degenerate quadratic space of dimension $\ell=n+2$ over $F$. We put $V_{\sigma_{i}}=V \otimes_{F, \sigma_{i}} \mathbb{R}$ and identify $V(\mathbb{R})=V \otimes_{\mathbb{Q}} \mathbb{R}=\bigoplus_{i} V_{\sigma_{i}}$. We assume that $V$ has signature

$$
((n, 2),(n+2,0), \ldots,(n+2,0)),
$$

that is, $V_{\sigma_{1}}$ has signature $(n, 2)$ and $V_{\sigma_{i}}$ has signature $(n+2,0)$ for $i=2, \ldots, d$. Sometimes we will also refer to the quantity

$$
\operatorname{sig}(V)=(n-2, n+2, \ldots, n+2) \in \mathbb{Z}^{d}
$$

as the signature. Let GSpin $(V)$ be the 'general' Spin group of $V$, that is, the group of all invertible elements $g$ in the even Clifford algebra of $V$ such that $g V g^{-1}=V$. It is an algebraic group over $F$, and the vector representation gives rise to an exact sequence

$$
1 \longrightarrow F^{\times} \longrightarrow \mathrm{GSpin}(V) \longrightarrow \mathrm{SO}(V) \longrightarrow 1 .
$$

We consider the algebraic group $H=\operatorname{Res}_{F / \mathbb{Q}} \operatorname{GSpin}(V)$ over $\mathbb{Q}$ given by Weil restriction of scalars. So $H(\mathbb{Q})$ can be identified with $\operatorname{GSpin}(V)(F)$.

We realize the hermitean symmetric space corresponding to $H$ as the Grassmannian $\mathbb{D}$ of oriented negative definite 2-dimensional subspaces of $V_{\sigma_{1}}$. Note that $\mathbb{D}$ has two components corresponding to the two possible choices of the orientation. The complex structure on $\mathbb{D}$ is most easily realized as follows. We let $V_{\mathbb{C}}=V \otimes_{F, \sigma_{1}} \mathbb{C}$ and extend the bilinear form $\mathbb{C}$-bilinearly to $V_{\mathbb{C}}$. The open subset

$$
\mathcal{K}=\left\{[Z] \in P\left(V_{\mathbb{C}}\right) ;(Z, Z)=0 \text { and }(Z, \bar{Z})<0\right\}
$$

of the zero quadric of the projective space $P\left(V_{\mathbb{C}}\right)$ of $V_{\mathbb{C}}$ is isomorphic to $\mathbb{D}$ by mapping $[Z]$ to the subspace $\mathbb{R} \Re(Z)+\mathbb{R} \Im(Z) \subset V_{\sigma_{1}}$ with the appropriate orientation.

We choose a pair $a, b \in V_{\sigma_{1}}$ of isotropic vectors such that $(a, b)=1$. The real quadratic space $V_{0}:=V_{\sigma_{1}} \cap a^{\perp} \cap b^{\perp}$ has signature $(n-1,1)$. The tube domain

$$
\mathcal{H}=\left\{z \in V_{0} \otimes_{\mathbb{R}} \mathbb{C} ; Q(\Im(z))<0\right\}
$$

is isomorphic to $\mathcal{K}$ by mapping $z \in \mathcal{H}$ to the class in $P\left(V_{\mathbb{C}}\right)$ of

$$
w(z)=z+a-Q(z) b .
$$

The domain $\mathcal{H}$ can be viewed as a generalized complex upper half plane. The linear action of $H(\mathbb{R})$ on $V_{\mathbb{C}}$ induces an action on $\mathcal{H}$ by fractional linear transformations. If $\gamma \in H(\mathbb{R})$, we have

$$
\gamma w(z)=j(\gamma, z) w(\gamma z)
$$

for an automorphy factor $j(\gamma, z)=(\gamma w(z), b)$.

The function

$$
Z \mapsto-\frac{1}{2}(Z, \bar{Z})=-(Y, Y)=:|Y|^{2}
$$


on $V_{\mathbb{C}}$ defines a hermitean metric on the tautological line bundle $\mathcal{L}$ over $\mathcal{K}$, where $Y=\Im(Z)$. Its first Chern form

$$
\Omega=-d d^{c} \log |Y|^{2}
$$

is $H(\mathbb{R})$-invariant and positive. It corresponds to an invariant Kähler metric on $\mathbb{D} \cong \mathcal{K}$ and gives rise to an invariant volume form $d \mu(z)=\Omega^{n}$. Note that for $z \in \mathcal{H}$ we have

$$
\begin{aligned}
-\frac{1}{2}(w(z), \overline{w(z)}) & =-(\Im(z), \Im(z)), \\
|\Im(\gamma z)|^{2} & =|j(\gamma, z)|^{-2}|\Im(z)|^{2} .
\end{aligned}
$$

For $K \subset H(\hat{\mathbb{Q}})$ compact open we consider the Shimura variety

$$
X_{K}=X_{K, V}:=H(\mathbb{Q}) \backslash(\mathbb{D} \times H(\hat{\mathbb{Q}})) / K .
$$

It is a quasi-projective variety of dimension $n$ defined over $F$. It is projective if and only if $V$ is anisotropic over $F$. By our assumption this is always the case when $d>1$. Let $\mathbb{D}^{+}$ be one of the two components of $\mathbb{D}$. The connected component of the identity $H(\mathbb{R})^{+}$of $H(\mathbb{R})$ acts on $\mathbb{D}^{+}$. We let $H(\mathbb{Q})^{+}=H(\mathbb{Q}) \cap H(\mathbb{R})^{+}$and write

$$
H(\hat{\mathbb{Q}})=\coprod_{j} H(\mathbb{Q})^{+} h_{j} K,
$$

as a finite disjoint union with $h_{j} \in H(\hat{\mathbb{Q}})$. Then we have

$$
X_{K}=\coprod_{j} \Gamma_{j} \backslash \mathbb{D}^{+},
$$

where $\Gamma_{j}=H(\mathbb{Q})^{+} \cap h_{j} K h_{j}^{-1}$. The different components of $X_{K}$ all have the same finite volume.

2.1. Modular forms. We define modular forms for the group $H(\mathbb{Q})$ as follows. A function $\Psi$ on $\mathcal{H} \times H(\hat{\mathbb{Q}})$ is called a meromorphic (holomorphic) modular form of weight $w \in \mathbb{Z}$ and level $K$ if:

(i) For fixed $h \in H(\hat{\mathbb{Q}})$ the function $\Psi(z, h)$ is meromorphic (holomorphic) in $z \in \mathcal{H}$,

(ii) $\Psi(z, h k)=\Psi(z, h)$ for all $k \in K$,

(iii) $\Psi(\gamma z, \gamma h)=j(\gamma, z)^{w} \Psi(z, h)$ for all $\gamma \in H(\mathbb{Q})$,

(iv) $\Psi$ is meromorphic (holomorphic) at the boundary.

The last condition is trivially fulfilled if $V$ is anisotropic over $F$. By the Koecher principle, it is also automatically fulfilled if the Witt rank of $V$ over $F$ (i.e. the dimension of a maximal totally isotropic subspace over $F$ ) is smaller than $n$. Using the decomposition (2.10), one can define functions $\Psi_{j}(z):=\Psi\left(z, h_{j}\right)$ on $\mathbb{D}^{+}$. They are classical modular forms of weight $w$ for the groups $\Gamma_{j}$. Consequently, the function $\Psi(z, h)$ corresponds to a tuple of classical modular forms $\left(\Psi_{j}\right)$.

The transformation law (iii) can be relaxed by allowing characters or multiplier systems. By slight abuse of notation, a function $\sigma: H(\mathbb{Q}) \times H(\hat{\mathbb{Q}}) \rightarrow \mathbb{C}^{\times}$is called a character for $H(\mathbb{Q})$, if $\sigma(\gamma, h k)=\sigma(\gamma, h)$ for all $k \in K$, and $\sigma\left(\gamma_{1} \gamma_{2}, h\right)=\sigma\left(\gamma_{1}, \gamma_{2} h\right) \sigma\left(\gamma_{2}, h\right)$ for all $\gamma_{1}, \gamma_{2} \in H(\mathbb{Q})$. Then the functions $\sigma_{j}(\gamma):=\sigma\left(\gamma, h_{j}\right)$ are homomorphisms $\Gamma_{j} \rightarrow \mathbb{C}^{\times}$. A 
function $\Psi$ on $\mathcal{H} \times H(\hat{\mathbb{Q}})$ is called a modular form of weight $w \in \mathbb{Z}$ and level $K$ with character $\sigma$, if it satisfies besides $(i),(i i),(i v)$ that

(iii') $\Psi(\gamma z, \gamma h)=\sigma(\gamma, h) j(\gamma, z)^{w} \Psi(z, h)$ for all $\gamma \in H(\mathbb{Q})$.

More generally, one can define modular forms of rational weight $w \in \mathbb{Q}$ with a multiplier system $H(\mathbb{Q}) \times H(\hat{\mathbb{Q}}) \rightarrow \mathbb{C}^{\times}$, see e.g. $[\mathrm{Br}]$, Chapter 3.3.

Modular forms of weight $w$ can be viewed as sections of the line bundle $\mathcal{M}_{w}$ of modular forms of weight $w$. The line bundle $\mathcal{M}_{1}$ of modular forms of weight 1 can be defined as the quotient

$$
H(\mathbb{Q}) \backslash \mathcal{L} \times H(\hat{\mathbb{Q}}) / K
$$

of the tautological bundle $\mathcal{L} \times H(\hat{\mathbb{Q}})$. The $w$-th power of this bundle is the line bundle of modular forms of weight $w$. The hermitean metric (2.5) on $\mathcal{L}$ induces a metric on the bundle $\mathcal{M}_{w}$ called the Petersson metric. For a modular form $\Psi$ of weight $w$ it is given by

$$
\|\Psi(z, h)\|_{P e t}=|\Psi(z, h)| \cdot|y|^{w} .
$$

The first Chern form of the line bundle $\mathcal{M}_{w}$ with the Petersson metric is $w \Omega$.

2.2. Lattices. Let $L \subset V$ be an $\mathcal{O}_{F}$-lattice, that is, a finitely generated $\mathcal{O}_{F}$-submodule such that $L \otimes_{\mathcal{O}_{F}} F=V$. We assume that $L$ is even, that is, $Q(L) \subset \partial^{-1}$. Then $Q_{\mathbb{Q}}(x)=$ $\operatorname{tr}_{F / \mathbb{Q}} Q(x)$ defines an even $\mathbb{Z}$-valued quadratic form on $L$. Let $L^{\prime}$ be the $\mathbb{Z}$-dual lattice of $L$ with respect to the quadratic form $Q_{\mathbb{Q}}$. The following is easily seen.

Proposition 2.1. We have

(1) $L^{\prime}$ is an $\mathcal{O}_{F}$-lattice.

(2) $L \subset L^{\prime}$ is a sublattice of finite index.

(3) $\left(L, L^{\prime}\right) \subset \partial^{-1}$.

The finite $\mathcal{O}_{F}$-module $L^{\prime} / L$ is called the discriminant group of $L$. We write $\hat{L}=L \otimes_{\mathbb{Z}} \hat{\mathbb{Z}}$, where $\hat{\mathbb{Z}}=\prod_{p} \mathbb{Z}_{p}$. We have $L^{\prime} / L \cong \hat{L}^{\prime} / \hat{L}$. The lattice $L$ is called unimodular if $L^{\prime}=L$.

Recall that $H(\hat{\mathbb{Q}})$ acts on the set of lattices $M \subset V$ by $M \mapsto h M:=(h \hat{M}) \cap V(F)$. This action induces an isomorphism $M^{\prime} / M \rightarrow(h M)^{\prime} /(h M)$, and $h M$ lies in the same genus as $M$. Throughout we assume that the compact open subgroup $K \subset H(\hat{\mathbb{Q}})$ fixes the lattice $L \subset V$ and acts trivially on $L^{\prime} / L$.

2.3. Special divisors. Here we define special divisors on $X_{K}$ (see e.g. [Ku1], [Bo1], [Br]). They generalize Heegner divisors on modular curves. We follow the description in [Ku1].

Let $x \in V$ be a vector of totally positive norm. We write $V_{x}$ for the orthogonal complement of $x$ in $V$ and $H_{x}$ for the stabilizer of $x$ in $H$. So $H_{x} \cong \operatorname{Res}_{F / \mathbb{Q}} \operatorname{GSpin}\left(V_{x}\right)$. The sub-Grassmannian

$$
\mathbb{D}_{x}=\{z \in \mathbb{D} ; z \perp x\}
$$

defines an analytic divisor on $\mathbb{D}$. For $h \in H(\hat{\mathbb{Q}})$ we consider the natural map

$$
H_{x}(\mathbb{Q}) \backslash \mathbb{D}_{x} \times H_{x}(\hat{\mathbb{Q}}) /\left(H_{x}(\hat{\mathbb{Q}}) \cap h K h^{-1}\right) \longrightarrow X_{K}, \quad\left(z, h_{1}\right) \mapsto\left(z, h_{1} h\right) .
$$


Its image defines a divisor $Z(x, h)$ on $X_{K}$, which is rational over $F$. Let $m \in F$ be totally positive, and let $\varphi \in S(V(\hat{F}))^{K}$ be a $K$-invariant Schwartz function. If there is an $x_{0} \in V(F)$ with $Q\left(x_{0}\right)=m$, we define the weighted cycle

$$
Z(m, \varphi)=\sum_{h \in H_{x_{0}}(\hat{\mathbb{Q}}) \backslash H(\hat{\mathbb{Q}}) / K} \varphi\left(h^{-1} x_{0}\right) Z\left(x_{0}, h\right) .
$$

The sum is finite, and $Z(m, \varphi)$ is a divisor on $X_{K}$ with complex coefficients. If there is no $x_{0} \in V(F)$ with $Q\left(x_{0}\right)=m$, we put $Z(m, \varphi)=0$. If $\mu \in L^{\prime} / L$ is a coset, and $\chi_{\mu}=\operatorname{char}(\mu+\hat{L}) \in S(V(\hat{F}))^{K}$ is the characteristic function, we briefly write

$$
Z(m, \mu):=Z\left(m, \chi_{\mu}\right) .
$$

\section{Theta Functions}

3.1. Special Schwartz functions. We begin by recalling some properties of Schwartz functions on quadratic spaces over $\mathbb{R}$. Let $\left(W, Q_{W}\right)$ be a real quadratic space of signature $(p, q)$ and write $S(W)$ for the space of Schwartz functions on $W$. Let $\mathbb{D}_{W}$ be the symmetric domain associated to $\mathrm{SO}(W)$, realized as the Grassmannian of $q$-dimensional negative definite oriented subspaces of $W$. When $q>0$, then $\mathbb{D}_{W}$ consists of two components corresponding to the two possible choices of the orientation, and when $q=0$, then $\mathbb{D}_{W}$ is a point.

Let $\mathbb{H}$ be the upper complex half plane. Let $\operatorname{Mp}_{2}(\mathbb{R})$ be the two-fold metaplectic cover of $\mathrm{SL}_{2}(\mathbb{R})$ realized by the two possible choices of a holomorphic square root of the automorphy factor $c \tau+d$ for $\left(\begin{array}{ll}a & b \\ c & d\end{array}\right) \in \mathrm{SL}_{2}(\mathbb{R})$. Recall that $\mathrm{Mp}_{2}(\mathbb{R})$ acts on $S(W)$ via the Weil representation $\omega_{W}$ associated to the standard additive character $u \mapsto e(u)$ of $\mathbb{R}$. Let $\widetilde{\mathrm{SO}}_{2}(\mathbb{R})$ be the inverse image of $\mathrm{SO}_{2}(\mathbb{R})$ in $\mathrm{Mp}_{2}(\mathbb{R})$, and let

$$
k_{\alpha}=\left(\begin{array}{cc}
\cos (\alpha) & \sin (\alpha) \\
-\sin (\alpha) & \cos (\alpha)
\end{array}\right) \in \mathrm{SO}_{2}(\mathbb{R})
$$

for $\alpha \in \mathbb{R}$. There is a character $\chi_{1 / 2}: \widetilde{\mathrm{SO}}_{2}(\mathbb{R}) \rightarrow \mathbb{C}^{\times}$given by

$$
\chi_{1 / 2}\left(k_{\alpha}, \phi\right) \mapsto \phi(i)^{-1}= \pm e^{i \alpha / 2} .
$$

We fix a base point $w_{0} \in \mathbb{D}_{W}$, and let $l \in \frac{1}{2} \mathbb{Z}$. Let $\varphi \in S(W)$ be a Schwartz function which is invariant under the stabilizer in $\mathrm{SO}(W)$ of $w_{0}$, and which is an eigenfunction of weight $l$ for $\widetilde{\mathrm{SO}}_{2}(\mathbb{R})$, that is, $\omega_{W}(\tilde{k})(\varphi)=\chi_{1 / 2}^{2 l}(\tilde{k}) \varphi$ for $\tilde{k} \in \widetilde{\mathrm{SO}}_{2}(\mathbb{R})$. Then we obtain a Schwartz function

$$
\varphi(\tau, w, \lambda)=\phi(i)^{2 l}\left(\omega_{W}\left(\tilde{g}_{\tau}, h_{w}\right) \varphi\right)(\lambda),
$$

depending on $\tau \in \mathbb{H}$ and $w \in \mathbb{D}_{W}$. Here $\tilde{g}_{\tau}=\left(g_{\tau}, \phi\right) \in \operatorname{Mp}_{2}(\mathbb{R})$ with $g_{\tau}(i)=\tau$ and $h_{w} \in \mathrm{SO}(W)$ with $h_{w} w_{0}=w$.

Every $w \in \mathbb{D}_{W}$ defines an orthogonal sum decomposition $W=w^{\perp} \oplus w$ into a positive definite subspace $w^{\perp}$ and a negative definite subspace $w$. If $\lambda \in W$ we write $\lambda_{w^{\perp}}$ and $\lambda_{w}$ for 
the corresponding orthogonal projections. The Gaussian associated to $w$ is the Schwartz function

$$
\varphi_{0}^{W}(w, \lambda)=\exp \left(-2 \pi Q_{W}\left(\lambda_{w^{\perp}}\right)+2 \pi Q_{W}\left(\lambda_{w}\right)\right) .
$$

It has weight $(p-q) / 2$. Using the explicit formulas for the Weil representation, it is easily seen that the corresponding Schwartz function $\varphi_{0}^{W}(\tau, w, \lambda)$ as in (3.3) is given by

$$
\varphi_{0}^{W}(\tau, w, \lambda)=\Im(\tau)^{q / 2} e\left(Q\left(\lambda_{w^{\perp}}\right) \tau+Q\left(\lambda_{w}\right) \bar{\tau}\right) .
$$

Kudla and Millson constructed a Schwartz form $\varphi_{K M}^{W}$ on $W$ taking values in $A^{q}\left(\mathbb{D}_{W}\right)$, the differential $q$-forms on $\mathbb{D}_{W}$ (see e.g. [KM3], and [BF] Section 4). We will require the following properties of this form. With respect to the natural action, we have the invariance

$$
\varphi_{K M}^{W}(w, \lambda) \in\left[A^{q}\left(\mathbb{D}_{W}\right) \otimes S(W)\right]^{\mathrm{SO}(W)},
$$

and $\varphi_{K M}^{W}(w, \lambda)$ is closed for all $\lambda \in W$. We have $\varphi_{K M}^{W}(w, \lambda)=P_{K M}(w, \lambda) \varphi_{0}^{W}(w, \lambda)$, where $P_{K M}(w, \lambda)$ is a polynomial on $W$ taking values in $A^{q}\left(\mathbb{D}_{W}\right)$. Under the action of the Weil representation, the function $\varphi_{K M}^{W}(\lambda)$ has weight $(p+q) / 2$. We also have the corresponding Schwartz function

$$
\varphi_{K M}^{W}(\tau, w, \lambda)=P_{K M}(w, \sqrt{\Im(\tau)} \lambda) e\left(Q\left(\lambda_{w^{\perp}}\right) \tau+Q\left(\lambda_{w}\right) \bar{\tau}\right) .
$$

When $q=0$, so that $W$ is positive definite, $\varphi_{K M}^{W}$ is simply the Gaussian $\varphi_{0}^{W}$.

In the present paper we only need the hermitean case $q=2$, for which a convenient construction of $\varphi_{K M}^{W}$ is given in [Ku3], Section 4. We have that $\varphi_{K M}^{W}(0)=-\Omega$, where $\Omega$ is the negative of the invariant Kähler form $\Omega$ on $\mathbb{D}_{W}$ defined in (2.6). The following relationship of $\varphi_{K M}^{W}$ and the Gaussian is obtained in [BF], Theorem 4.4.

Proposition 3.1. Assume that $q=2$ so that $\mathbb{D}_{W}$ is hermitean. We have

$$
d d^{c} \varphi_{0}^{W}(\tau, w, \lambda)=-L \varphi_{K M}^{W}(\tau, w, \lambda),
$$

where $L=-2 i \Im(\tau)^{2} \frac{\partial}{\partial \bar{\tau}}$ denotes the Maass lowering operator, and $d$ and $d^{c}=\frac{1}{4 \pi i}(\partial-\bar{\partial})$ are the usual differentials on $\mathbb{D}_{W}$.

3.2. Discriminant forms and the Weil representation. We now come back to our global quadratic space $V$ over the totally real field $F$ as in Section 2, Let $\mathbb{H}$ be the upper complex half plane. We use $\tau=\left(\tau_{1}, \ldots, \tau_{d}\right)$ as a standard variable on $\mathbb{H}^{d}$ and put $u_{i}=\Re\left(\tau_{i}\right), v_{i}=\Im\left(\tau_{i}\right)$. For a $d$-tuple $\left(w_{1}, \ldots, w_{d}\right)$ of complex numbers, we put $\operatorname{tr}(w)=\sum_{i} w_{i}$ and $\mathrm{N}(w)=\prod_{i} w_{i}$.

We view $\mathbb{C}^{d}$ as a $\mathbb{R}^{d}$-module by putting $\lambda w=\left(\lambda_{1} w_{1}, \ldots, \lambda_{d} w_{d}\right)$ for $\lambda=\left(\lambda_{1}, \ldots, \lambda_{d}\right) \in \mathbb{R}^{d}$. For $x \in F$ we briefly write $x_{i}=\sigma_{i}(x)$ and identify $x$ with its image $\left(x_{1}, \ldots, x_{d}\right) \in \mathbb{R}^{d}$. The usual trace and norm of $x$ coincide with the above definitions. Moreover, the inclusion $F \rightarrow \mathbb{R}^{d}$ defines an $F$-vector space structure on $\mathbb{C}^{d}$.

We are interested in certain vector valued modular forms for the Hilbert modular group associated to $F$. Let $G=\operatorname{Res}_{F / \mathbb{Q}} \mathrm{SL}_{2}$. For $g \in \mathrm{SL}_{2}(F) \cong G(\mathbb{Q})$, we briefly write $g_{i}=\sigma_{i}(g)$. So the image of $g$ in $G(\mathbb{R}) \cong \mathrm{SL}_{2}(\mathbb{R})^{d}$ is given by $\left(g_{1}, \ldots, g_{d}\right)$. The group $G(\mathbb{R})$ acts on $\mathbb{H}^{d}$ by fractional linear transformations. We denote by $\tilde{G}_{\mathbb{A}}$ the twofold metaplectic cover of 
$G(\mathbb{A})$. Let $\tilde{G}_{\mathbb{R}}$ be the full inverse image in $\tilde{G}_{\mathbb{A}}$ of $G(\mathbb{R})$. We will frequently realize $\tilde{G}_{\mathbb{R}}$ as the group of pairs

$$
(g, \phi(\tau))
$$

where $g=\left(\begin{array}{ll}a & b \\ c & d\end{array}\right) \in G(\mathbb{R})$ and $\phi(\tau)$ is a holomorphic function on $\mathbb{H}^{d}$ such that $\phi(\tau)^{2}=$ $\mathrm{N}(c \tau+d)$. The product of $\left(g_{1}, \phi_{1}(\tau)\right),\left(g_{2}, \phi_{2}(\tau)\right)$ is given by

$$
\left(g_{1}, \phi_{1}(\tau)\right)\left(g_{2}, \phi_{2}(\tau)\right)=\left(g_{1} g_{2}, \phi_{1}\left(g_{2} \tau\right) \phi_{2}(\tau)\right) .
$$

Let $\tilde{\Gamma}$ be the full inverse image in $\tilde{G}_{\mathbb{R}}$ of the Hilbert modular group

$$
\Gamma=\mathrm{SL}_{2}\left(\mathcal{O}_{F}\right) \subset G(\mathbb{R}) .
$$

It follows from Vaserstein's theorem that $\tilde{\Gamma}$ is generated by the elements

$$
\begin{aligned}
T_{b} & =\left(\left(\begin{array}{ll}
1 & b \\
0 & 1
\end{array}\right), 1\right), \quad b \in \mathcal{O}_{F}, \\
S & =\left(\left(\begin{array}{cc}
0 & -1 \\
1 & 0
\end{array}\right), \sqrt{\mathrm{N}(\tau)}\right), \\
N & =\left(\left(\begin{array}{ll}
1 & 0 \\
0 & 1
\end{array}\right),-1\right) .
\end{aligned}
$$

We also put $Z=\left(\left(\begin{array}{cc}-1 & 0 \\ 0 & -1\end{array}\right), i^{d}\right)$. Observe that we have the relations $(S T)^{3}=S^{2}=Z$. If $d$ is odd then $Z^{2}=N$, and if $d$ is even then $Z^{2}=N^{2}=1$. There are further relations corresponding to the elliptic fixed points of $\Gamma$.

Let $L \subset V$ be an $\mathcal{O}_{F}$-lattice. For $\mu \in L^{\prime} / L$ we write $\chi_{\mu}=\operatorname{char}(\mu+\hat{L}) \in S(V(\hat{F}))$ for the characteristic function of the coset. Associated to the reductive dual pair $\left(\mathrm{SL}_{2}, \mathrm{O}(V)\right)$ there is a Weil representation $\omega=\omega_{\psi}$ of $\tilde{G}_{\mathbb{A}}$ on the Schwartz space $S\left(V\left(\mathbb{A}_{F}\right)\right)$, where $\psi$ is the standard additive character of $F \backslash \mathbb{A}_{F}$ with $\psi_{\infty}(x)=e(\operatorname{tr} x)$ [We1]. The subspace

$$
S_{L}=\bigoplus_{\mu \in L^{\prime} / L} \mathbb{C} \chi_{\mu} \subset S(V(\hat{F}))
$$

is preserved by the action of $\widetilde{\mathrm{SL}}_{2}\left(\hat{\mathcal{O}}_{F}\right)$, the full inverse image in $\tilde{G}_{\mathbb{A}}$ of $\mathrm{SL}_{2}\left(\hat{\mathcal{O}}_{F}\right) \subset G(\hat{\mathbb{Q}})$. The canonical splitting $G(F) \rightarrow \tilde{G}_{\mathbb{A}}$ defines a homomorphism

$$
\tilde{\Gamma} \longrightarrow \widetilde{\mathrm{SL}}_{2}\left(\hat{\mathcal{O}}_{F}\right), \quad \gamma \mapsto \hat{\gamma}
$$

where $\hat{\gamma}$ is the unique element such that $\gamma \hat{\gamma}$ is in the image of $G(F)$. This induces a representation $\rho_{L}$ of $\tilde{\Gamma}$ on $S_{L}$ by

$$
\rho_{L}(\gamma) \varphi=\bar{\omega}(\hat{\gamma}), \quad \gamma \in \tilde{\Gamma} .
$$

In terms of the above generators of $\tilde{\Gamma}$ the representation $\rho_{L}$ is given by

$$
\begin{aligned}
\rho_{L}\left(T_{b}\right)\left(\chi_{\mu}\right) & =e(\operatorname{tr}(Q(\mu) b)) \chi_{\mu}, \quad b \in \mathcal{O}_{F}, \\
\rho_{L}(S)\left(\chi_{\mu}\right) & =\frac{e(-\operatorname{tr}(\operatorname{sig} V) / 8)}{\sqrt{\left|L^{\prime} / L\right|}} \sum_{\nu \in L^{\prime} / L} e(-\operatorname{tr}(\mu, \nu)) \chi_{\nu}, \\
\rho_{L}(N)\left(\chi_{\mu}\right) & =(-1)^{\ell} \chi_{\mu} .
\end{aligned}
$$


Note that this is compatible with the conventions in [Bo1], [Ku3], [Br], where the case $F=\mathbb{Q}$ is considered. We also have the useful formula $\rho_{L}(Z)\left(\chi_{\mu}\right)=e(-\operatorname{tr}(\operatorname{sig} V) / 4) \chi_{-\mu}$. For a totally positive unit $\varepsilon \in \mathcal{O}_{F}^{\times}$the element $m(\varepsilon)=\left(\left(\begin{array}{cc}\varepsilon & 0 \\ 0 & \varepsilon^{-1}\end{array}\right), 1\right)$ acts by

$$
\rho_{L}(m(\varepsilon))\left(\chi_{\mu}\right)=\chi_{\varepsilon^{-1} \mu} \text {. }
$$

We denote the standard $\mathbb{C}$-bilinear pairing on $S_{L}$ (the $L^{2}$ bilinear pairing) by

$$
\langle a, b\rangle=\sum_{\mu \in L^{\prime} / L} a_{\mu} b_{\mu}
$$

for $a, b \in S_{L}$. The representation $\rho_{L}$ is unitary, that is, we have $\left\langle\bar{\rho}_{L} a, \rho_{L} b\right\rangle=\langle a, b\rangle$. It is well known that $\rho_{L}$ factors through a finite quotient of $\tilde{\Gamma}$.

3.3. Siegel theta functions. Next, we define Siegel theta functions for the lattice $L$. For the global quadratic space $V$ over $F$, we obtain the Gaussian on $V(\mathbb{R})$ by piecing together the local data. For $\lambda=\left(\lambda_{1}, \ldots, \lambda_{d}\right) \in V(\mathbb{R})$ and $z \in \mathbb{D}$ we define

$$
\varphi_{0}(z, \lambda)=\varphi_{0}^{V_{\sigma_{1}}}\left(z, \lambda_{1}\right) \otimes \varphi_{0}^{V_{\sigma_{2}}}\left(\lambda_{2}\right) \otimes \cdots \otimes \varphi_{0}^{V_{\sigma_{d}}}\left(\lambda_{d}\right) .
$$

By our assumption on the signature of $V$, it has weight $\left(\frac{n-2}{2}, \frac{n+2}{2}, \ldots, \frac{n+2}{2}\right)$. We obtain the corresponding Schwartz function

$$
\varphi_{0}(\tau, z, \lambda)=\varphi_{0}^{V_{\sigma_{1}}}\left(\tau_{1}, z, \lambda_{1}\right) \otimes \varphi_{0}^{V_{\sigma_{2}}}\left(\tau_{2}, \lambda_{2}\right) \otimes \cdots \otimes \varphi_{0}^{V_{\sigma_{d}}}\left(\tau_{d}, \lambda_{d}\right)
$$

for $\tau \in \mathbb{H}^{d}$. It can be explicitly described as follows. We put

$$
Q(\lambda)=\left(Q\left(\lambda_{1}\right), \ldots, Q\left(\lambda_{d}\right)\right) \in \mathbb{R}^{d} .
$$

If $z \in \mathbb{D}$ we let $\lambda_{1 z}$ (respectively $\lambda_{1 z^{\perp}}$ ) be the orthogonal projection of $\lambda_{1}$ to $z$ (respectively to $\left.z^{\perp}\right)$. Moreover, we put

$$
\begin{aligned}
\lambda_{z} & =\left(\lambda_{1 z}, 0, \ldots, 0\right), \\
\lambda_{z^{\perp}} & =\left(\lambda_{1 z^{\perp}}, \lambda_{2}, \ldots, \lambda_{d}\right) .
\end{aligned}
$$

Hence

$$
\lambda \mapsto \operatorname{tr} Q\left(\lambda_{z^{\perp}}\right)-\operatorname{tr} Q\left(\lambda_{z}\right)
$$

defines a positive definite quadratic form on $V(\mathbb{R})$, the majorant associated to $z$. It is easily seen that

$$
\varphi_{0}(\tau, z, \lambda)=v_{1} e\left(\operatorname{tr} Q\left(\lambda_{z^{\perp}}\right) \tau+\operatorname{tr} Q\left(\lambda_{z}\right) \bar{\tau}\right) .
$$

In particular, this Schwartz function is non-holomorphic in $\tau_{1}$, but holomorphic in $\tau_{2}, \ldots, \tau_{d}$.

We identify $V(F)$ with its image under the canonical embedding into $V(\mathbb{R})$. Let $\varphi_{f} \in$ $S(V(\hat{F}))$ be a Schwartz-Bruhat function. For $\tau \in \mathbb{H}^{d}, z \in \mathbb{D}$, and $h \in H(\hat{\mathbb{Q}})$, we define the Siegel theta function associated to $\varphi_{f}$ by

$$
\begin{aligned}
\theta_{S}\left(\tau, z, h ; \varphi_{f}\right) & =\sum_{\lambda \in V(F)} \varphi_{f}\left(h^{-1} \lambda\right) \varphi_{0}(\tau, z, \lambda) \\
& =v_{1} \sum_{\lambda \in V(F)} \varphi_{f}\left(h^{-1} \lambda\right) e\left(\operatorname{tr} Q\left(\lambda_{z^{\perp}}\right) \tau+\operatorname{tr} Q\left(\lambda_{z}\right) \bar{\tau}\right) .
\end{aligned}
$$


It satisfies the transformation formulas

$$
\begin{aligned}
\theta_{S}\left(\tau, \gamma z, \gamma h ; \varphi_{f}\right) & =\theta_{S}\left(\tau, z, h ; \varphi_{f}\right), \quad \gamma \in H(\mathbb{Q}), \\
\theta_{S}\left(\gamma \tau, z, h ; \varphi_{f}\right) & =\left(c_{1} \tau_{1}+d_{1}\right)^{-2} \phi(\tau)^{\ell} \theta_{S}\left(\tau, z, h ; \omega\left(\hat{\gamma}^{-1}\right) \varphi_{f}\right), \quad \gamma=\left(\left(\begin{array}{ll}
a & b \\
c & d
\end{array}\right), \phi\right) \in \tilde{\Gamma} .
\end{aligned}
$$

We will also consider the $S_{L}$-valued theta function

$$
\Theta_{S}(\tau, z, h)=\sum_{\mu \in L^{\prime} / L} \theta_{S}\left(\tau, z, h ; \chi_{\mu}\right) \chi_{\mu} .
$$

It has the following transformation formula, which can be deduced from (3.12) or by applying the Poisson summation formula as in [Bo1].

Theorem 3.2. For $\gamma=(g, \phi(\tau)) \in \tilde{\Gamma}$ with $g=\left(\begin{array}{ll}a & b \\ c & d\end{array}\right)$, we have

$$
\Theta_{S}(\gamma \tau, z, h)=\left(c_{1} \tau_{1}+d_{1}\right)^{-2} \phi(\tau)^{\ell} \rho_{L}(\gamma) \Theta_{S}(\tau, z, h) .
$$

The following growth estimate will be used later. It is proved in the same way as the corresponding statement for holomorphic Hilbert modular forms.

Proposition 3.3. The Siegel theta function satisfies uniformly in $u$ that

$$
\theta_{S}\left(\tau, z, h ; \chi_{\mu}\right)=O\left(v_{1} N(v)^{-(n+2) / 2}\right), \quad v_{i} \rightarrow 0 .
$$

3.4. Kudla-Millson theta functions. For $\lambda=\left(\lambda_{1}, \ldots, \lambda_{d}\right) \in V(\mathbb{R})$ and $z \in \mathbb{D}$ the Kudla-Millson Schwartz form on $V(\mathbb{R})$ is defined by

$$
\varphi_{K M}(z, \lambda)=\varphi_{K M}^{V_{\sigma_{1}}}\left(z, \lambda_{1}\right) \otimes \varphi_{K M}^{V_{\sigma_{2}}}\left(\lambda_{2}\right) \otimes \cdots \otimes \varphi_{K M}^{V_{\sigma_{d}}}\left(\lambda_{d}\right) .
$$

It has parallel weight $\ell / 2=(n+2) / 2$. We also have the corresponding Schwartz form $\varphi_{K M}(\tau, z, \lambda)$ for $\tau \in \mathbb{H}^{d}$. It is non-holomorphic in $\tau_{1}$, but holomorphic in $\tau_{2}, \ldots, \tau_{d}$.

Let $\varphi_{f} \in S(V(\hat{F}))$ be a Schwartz-Bruhat function. For $\tau \in \mathbb{H}^{d}, z \in \mathbb{D}$, and $h \in H(\hat{\mathbb{Q}})$, the Kudla-Millson theta function associated to $\varphi_{f}$ is given by

$$
\theta_{K M}\left(\tau, z, h ; \varphi_{f}\right)=\sum_{\lambda \in V(F)} \varphi_{f}\left(h^{-1} \lambda\right) \varphi_{K M}(\tau, z, \lambda)
$$

see [KM1], [KM2], [KM3] for details. Its geometric significance lies in the fact that the Fourier coefficients with totally positive index $m$ are Poincaré dual forms for the cycles $Z\left(m, \varphi_{f}\right)$ (however, we do not need that here).

The characteristic functions of the cosets of $L$ can be used to define the $S_{L}$-valued valued Kudla-Millson theta function

$$
\Theta_{K M}(\tau, z, h)=\sum_{\mu \in L^{\prime} / L} \theta_{K M}\left(\tau, z, h ; \chi_{\mu}\right) \chi_{\mu} .
$$

In the variable $(z, h)$ it defines a closed 2 -form on $X_{K}$. In $\tau$ it satisfies the transformation formula

$$
\Theta_{K M}(\gamma \tau, z, h)=\phi(\tau)^{\ell} \rho_{L}(\gamma) \Theta_{K M}(\tau, z, h)
$$


for $\gamma=(g, \phi(\tau)) \in \tilde{\Gamma}$. Hence it is a non-holomorphic vector valued Hilbert modular form for $\tilde{\Gamma}$ of parallel weight $\ell / 2$ with representation $\rho_{L}$. Since it has moderate growth at the cusps, it can be integrated against cusp forms.

Proposition 3.4. The Kudla-Millson theta function satisfies uniformly in $u$ that

$$
\theta_{K M}\left(\tau, z, h ; \chi_{\mu}\right)=O\left(N(v)^{-(n+2) / 2}\right), \quad v_{i} \rightarrow 0 .
$$

\section{WHITTAKER FORMS}

Following Harvey, Moore, and Borcherds (see [HM] and [Bo1]) we would like to construct automorphic forms on $X_{K}$ with singularities along special cycles by integrating weakly holomorphic modular forms or weak Maass forms against the Siegel theta function. Unfortunately, because of the Koecher principle, there are no such automorphic forms with singularities at the cusps when $d>1$.

Here we overcome this problem by viewing weak Maass forms as formal Poincaré series ignoring the issue of convergence. Then the theta integral can be formally unfolded leading to an integral over $\tilde{\Gamma}_{\infty} \backslash \mathbb{H}^{d}$, where

$$
\tilde{\Gamma}_{\infty}:=\left\{T_{b} \cdot(1, \pm 1) ; \quad b \in \mathcal{O}_{F}\right\}
$$

is the subgroup of translations of $\tilde{\Gamma}$. It turns out that such integrals still make sense when they are suitably regularized. It is natural to consider them for translation invariant functions which are eigenfunctions for the Laplacians. This leads to the definition of Whittaker forms below. Moreover, we define weakly holomorphic Whittaker forms, which serve as substitutes of weakly holomorphic modular forms. We derive some properties of these functions which will be important later.

We begin by fixing some notation. Let $k=\left(k_{1}, \ldots, k_{d}\right) \in\left(\frac{1}{2} \mathbb{Z}\right)^{d}$ be a weight. Throughout we assume that $k \equiv\left(\frac{\ell}{2}, \ldots, \frac{\ell}{2}\right)\left(\bmod \mathbb{Z}^{d}\right)$. We define a Petersson slash operator in weight $k$ for the representation $\rho_{L}$ on functions $f: \mathbb{H}^{d} \rightarrow S_{L}$ by

$$
\left(\left.f\right|_{k, \rho_{L}}(g, \phi)\right)(\tau)=\left(c_{1} \tau_{1}+d_{1}\right)^{-k_{1}+\ell / 2} \cdots\left(c_{d} \tau_{d}+d_{d}\right)^{-k_{d}+\ell / 2} \phi(\tau)^{-\ell} \rho_{L}(g, \phi)^{-1} f(g \tau),
$$

where $(g, \phi) \in \tilde{G}_{\mathbb{R}}$ and $g=\left(\begin{array}{ll}a & b \\ c & d\end{array}\right)$. The Petersson slash operator for the dual representation $\bar{\rho}_{L}$ is defined analogously. We write $S_{k, \rho_{L}}$ for the space of vector valued Hilbert cusp forms of weight $k$ for $\tilde{\Gamma}$ with representation $\rho_{L}$.

We have the usual hyperbolic Laplace operators in weight $k$ acting on smooth functions on $\mathbb{H}^{d}$. They are given by

$$
\Delta_{k}^{(j)}=-v_{j}^{2}\left(\frac{\partial^{2}}{\partial u_{j}^{2}}+\frac{\partial^{2}}{\partial v_{j}^{2}}\right)+i k_{j} v_{j}\left(\frac{\partial}{\partial u_{j}}+i \frac{\partial}{\partial v_{j}}\right)
$$

for $j=1, \ldots, d$. Moreover, we have the Maass lowering and raising operators

$$
\begin{aligned}
R_{k}^{(j)} & =2 i \frac{\partial}{\partial \tau_{j}}+k_{j} v_{j}^{-1}, \\
L_{k}^{(j)} & =-2 i v_{j}^{2} \frac{\partial}{\partial \bar{\tau}_{j}} .
\end{aligned}
$$


The raising operator $R_{k}^{(j)}$ raises the weight of an automorphic form in the $j$-th component by 2 while $L_{k}^{(j)}$ lowers it by 2 . The Laplacian $\Delta_{k}^{(j)}$ satisfies the identity

$$
-\Delta_{k}^{(j)}=L_{k+2}^{(j)} R_{k}^{(j)}+k_{j}=R_{k-2}^{(j)} L_{k}^{(j)} .
$$

We consider functions which are invariant under the group of translations $\tilde{\Gamma}_{\infty}$. For $s \in \mathbb{C}$ we let $A_{k, \bar{\rho}_{L}}(s)$ be the space of smooth functions $f: \mathbb{H}^{d} \rightarrow S_{L}$ satisfying:

(1) $f\left(T_{b} \tau\right)=\bar{\rho}_{L}\left(T_{b}\right) f(\tau), \quad b \in \mathcal{O}_{F}$,

(2) $\Delta_{k}^{(1)} f=\frac{1}{4}\left(k_{1}-1+s\right)\left(k_{1}-1-s\right) f$,

(3) $f$ is antiholomorphic in $\tau_{j}$ for $j=2, \ldots, d$.

To describe the Fourier expansion of such a function we recall some properties of Whittaker functions, see [AbSt Chap. 13 pp. 189 or [Er1 Vol. I Chap. 6 p. 264. Kummer's confluent hypergeometric function is defined by

$$
M(a, b, z)=\sum_{n=0}^{\infty} \frac{(a)_{n}}{(b)_{n}} \frac{z^{n}}{n !},
$$

where $(a)_{n}=\Gamma(a+n) / \Gamma(a)$ and $(a)_{0}=1$. The Whittaker functions are defined by

$$
\begin{aligned}
& M_{\nu, \mu}(z)=e^{-z / 2} z^{1 / 2+\mu} M(1 / 2+\mu-\nu, 1+2 \mu, z), \\
& W_{\nu, \mu}(z)=\frac{\Gamma(-2 \mu)}{\Gamma(1 / 2-\mu-\nu)} M_{\nu, \mu}(z)+\frac{\Gamma(2 \mu)}{\Gamma(1 / 2+\mu-\nu)} M_{\nu,-\mu}(z) .
\end{aligned}
$$

They are linearly independent solutions of the Whittaker differential equation. The $M$ Whittaker function has the asymptotic behavior

$$
\begin{aligned}
& M_{\nu, \mu}(z)=z^{\mu+1 / 2}(1+O(z)), \quad z \rightarrow 0, \\
& M_{\nu, \mu}(z)=\frac{\Gamma(1+2 \mu)}{\Gamma(\mu-\nu+1 / 2)} e^{z / 2} z^{-\nu}\left(1+O\left(z^{-1}\right)\right), \quad z \rightarrow \infty,
\end{aligned}
$$

while $W_{\nu, \mu}(z)$ is exponentially decreasing for real $z \rightarrow \infty$ and behaves like a constant times $z^{-\mu+1 / 2}$ as $z \rightarrow 0$.

For convenience we put for $s \in \mathbb{C}$ and $v_{1} \in \mathbb{R}$ :

$$
\begin{aligned}
& \mathcal{M}_{s}\left(v_{1}\right)=\left|v_{1}\right|^{-k_{1} / 2} M_{\operatorname{sgn}\left(v_{1}\right) k_{1} / 2, s / 2}\left(\left|v_{1}\right|\right) \cdot e^{-v_{1} / 2}, \\
& \mathcal{W}_{s}\left(v_{1}\right)=\left|v_{1}\right|^{-k_{1} / 2} W_{\operatorname{sgn}\left(v_{1}\right) k_{1} / 2, s / 2}\left(\left|v_{1}\right|\right) \cdot e^{-v_{1} / 2} .
\end{aligned}
$$

The functions $\mathcal{M}_{s}\left(v_{1}\right)$ and $\mathcal{W}_{s}\left(v_{1}\right)$ are holomorphic in $s$. Later we will be interested in their special value at

$$
s_{0}=1-k_{1} .
$$

We have

$$
\begin{aligned}
& \mathcal{M}_{s_{0}}\left(v_{1}\right)=\left(-\operatorname{sgn}\left(v_{1}\right)\right)^{k_{1}-1} \cdot e^{-v_{1}}\left(\Gamma\left(2-k_{1}\right)-\left(1-k_{1}\right) \Gamma\left(1-k_{1},-v_{1}\right)\right), \\
& \mathcal{W}_{s_{0}}\left(v_{1}\right)= \begin{cases}e^{-v_{1}}, & v_{1}>0, \\
e^{-v_{1}} \cdot \Gamma\left(1-k_{1},-v_{1}\right), & v_{1}<0,\end{cases}
\end{aligned}
$$


where $\Gamma(a, z)$ denotes the incomplete Gamma function. Any $f \in A_{k, \bar{\rho}_{L}}(s)$ has a Fourier expansion of the form

$$
\begin{aligned}
f(\tau)= & \sum_{\substack{\mu \in L^{\prime} / L \\
Q(\mu) \in \partial_{F}^{-1}}}\left(a(0, \mu, s) v_{1}^{\left(1-k_{1}-s\right) / 2}+b(0, \mu, s) v_{1}^{\left(1-k_{1}+s\right) / 2}\right) \chi_{\mu} \\
& +\sum_{\mu \in L^{\prime} / L} \sum_{\substack{m \in \partial_{F}^{-1}-Q(\mu) \\
m \neq 0}}\left(a(m, \mu, s) \mathcal{W}_{s}\left(4 \pi m_{1} v_{1}\right)+b(m, \mu, s) \mathcal{M}_{s}\left(4 \pi m_{1} v_{1}\right)\right) e(\operatorname{tr}(m \bar{\tau})) \chi_{\mu} .
\end{aligned}
$$

Special elements of $A_{k, \bar{\rho}_{L}}(s)$ are the functions

$$
f_{m, \mu}(\tau, s):=C(m, k, s) \mathcal{M}_{s}\left(-4 \pi m_{1} v_{1}\right) e(-\operatorname{tr}(m \bar{\tau})) \chi_{\mu}
$$

for $\mu \in L^{\prime} / L, m \in \partial^{-1}+Q(\mu)$, and $m \gg 0$. Here $C(m, k, s)$ denotes the normalizing factor

$$
C(m, k, s):=\frac{\left(4 \pi m_{2}\right)^{k_{2}-1} \cdots\left(4 \pi m_{d}\right)^{k_{d}-1}}{\Gamma(s+1) \Gamma\left(k_{2}-1\right) \cdots \Gamma\left(k_{d}-1\right)},
$$

which turns out to be convenient later (for instance in Proposition 4.5).

Definition 4.1. A Whittaker form of weight $k$ and parameter $s$ (for $\tilde{\Gamma}$ and $\bar{\rho}_{L}$ ) is a finite linear combination of the functions $f_{m, \mu}(\tau, s)$ for $\mu \in L^{\prime} / L, m \in \partial_{F}^{-1}+Q(\mu)$, and $m \gg 0$. A harmonic Whittaker form is a Whittaker form with parameter $s_{0}$. We denote the $\mathbb{C}$-vector space of such harmonic Whittaker forms by $H_{k, \bar{\rho}_{L}}$.

So a harmonic Whittaker form is a finite linear combination of the functions

$$
f_{m, \mu}(\tau):=f_{m, \mu}\left(\tau, s_{0}\right)
$$

for $\mu \in L^{\prime} / L, m \in \partial_{F}^{-1}+Q(\mu)$, and $m \gg 0$. Explicitly we have

$$
f_{m, \mu}(\tau)=C\left(m, k, s_{0}\right) \Gamma\left(2-k_{1}\right)\left(1-\frac{\Gamma\left(1-k_{1}, 4 \pi m_{1} v_{1}\right)}{\Gamma\left(1-k_{1}\right)}\right) e^{4 \pi m_{1} v_{1}} e(\operatorname{tr}(-m \bar{\tau})) \chi_{\mu} .
$$

We define the dual weight for $k$ by $\kappa=\left(2-k_{1}, k_{2}, \ldots, k_{d}\right)$. We consider the differential operator $\delta_{k}$ on functions $f: \mathbb{H}^{d} \rightarrow S_{L}$ given by

$$
\delta_{k}(f)=v_{1}^{k_{1}-2} \overline{L_{k}^{(1)} f(\tau)} .
$$

If $f \in H_{k, \bar{\rho}_{L}}$, then $\delta_{k}(f)$ is a holomorphic function satisfying $f\left(T_{b} \tau\right)=\rho_{L}\left(T_{b}\right) f(\tau)$ for all $b \in \mathcal{O}_{F}$. In particular, we have

$$
\delta_{k}\left(f_{m, \mu}\right)(\tau)=\frac{\left(4 \pi m_{1}\right)^{\kappa_{1}-1} \cdots\left(4 \pi m_{d}\right)^{\kappa_{d}-1}}{\Gamma\left(\kappa_{1}-1\right) \cdots \Gamma\left(\kappa_{d}-1\right)} e(\operatorname{tr}(m \tau)) \chi_{\mu} .
$$

We have $\Delta_{k}^{(1)} f=0$ for a harmonic Whittaker form $f$. The functions $f_{m, \mu}\left(\tau,-s_{0}\right)$ have eigenvalue zero for this Laplacian as well, but they are not harmonic Whittaker forms in the sense of our definition. The point of the definition is to prescribe a particular growth as $v_{j} \rightarrow \infty$ and $v_{j} \rightarrow 0$ such that there is a smooth relationship with Hilbert cusp forms (see below) and such that the theta lift of a harmonic Whittaker form has singularities along special divisors (see Section 5). More precisely, we have the following characterization: 
Remark 4.2. Assume that $k_{1}<1$ (which will be the case in all later applications). Then $H_{k, \bar{\rho}_{L}}$ is the space of functions in $A_{k, \bar{\rho}_{L}}\left(s_{0}\right)$ satisfying:

(4) only finitely many Fourier coefficients are non-zero,

(5) there is a totally positive $\varepsilon \in F$ such that $\delta_{k}(f)(\tau)=O\left(e^{-\operatorname{tr}(\varepsilon v)}\right)$ for $v_{j} \rightarrow \infty$,

(6) we have $f(\tau)=O\left(v_{1}^{s_{0}}\right)$, for $v_{1} \rightarrow 0$.

For the rest of this section we assume that $\kappa_{j} \geq 3 / 2$ for $j=1, \ldots, d$. We define an operator $\xi_{k}: H_{k, \bar{\rho}_{L}} \rightarrow S_{\kappa, \rho_{L}}$ by

$$
\xi_{k}(f)=\left.\sum_{\gamma \in \tilde{\Gamma}_{\infty} \backslash \tilde{\Gamma}} \delta_{k}(f)\right|_{\kappa, \rho_{L}} \gamma
$$

When $\kappa_{j}>2$ for $j=1, \ldots, d$, the Poincaré series on the right hand side converges normally by a standard estimate (see e.g. [Ga], Chapter 1.13) and defines a holomorphic cusp form. When $\kappa_{j} \geq 3 / 2$ we define the Poincaré series using "Hecke summation" as the value at $s^{\prime}=0$ of the holomorphic continuation in $s^{\prime}$ of

$$
\left.\sum_{\gamma \in \tilde{\Gamma}_{\infty} \backslash \tilde{\Gamma}} \delta_{k}(f) \mathrm{N}(v)^{s^{\prime}}\right|_{\kappa, \rho_{L}} \gamma .
$$

Proposition 4.3. Assume that $\kappa_{j} \geq 3 / 2$ for $j=1, \ldots, d$. The $\operatorname{map} \xi_{k}: H_{k, \bar{\rho}_{L}} \rightarrow S_{\kappa, \rho_{L}}$ is surjective.

Proof. The assertion follows from (4.17) and the fact that $S_{\kappa, \rho_{L}}$ is generated by Poincaré series.

Definition 4.4. A Whittaker form $f$ is called weakly holomorphic if it is harmonic and satisfies $\xi_{k}(f)=0$. We denote by $M_{k, \bar{\rho}_{L}}^{!}$the subspace of weakly holomorphic Whittaker forms in $H_{k, \bar{\rho}_{L}}$.

Note that a weakly holomorphic Whittaker form is not holomorphic as a function on $\mathbb{H}^{d}$. It is rather holomorphic in a weak distribution sense. In view of Proposition 4.3, we have the exact sequence

$$
0 \longrightarrow M_{k, \bar{\rho}_{L}}^{!} \longrightarrow H_{k, \bar{\rho}_{L}} \stackrel{\xi_{k}}{\longrightarrow} S_{\kappa, \rho_{L}} \longrightarrow 0 .
$$

Recall that the Petersson scalar product of $f, g \in S_{\kappa, \rho_{L}}$ is given by

$$
(f, g)_{P e t}=\frac{1}{\sqrt{D}} \int_{\tilde{\Gamma} \backslash \mathbb{H}^{d}}\langle f, \bar{g}\rangle v^{\kappa} d \mu(\tau),
$$

where $d \mu(\tau)=\frac{d u_{1} d v_{1}}{v_{1}^{2}} \ldots \frac{d u_{d} d v_{d}}{v_{d}^{2}}$ is the invariant measure on $\mathbb{H}^{d}$, and $v^{\kappa}$ is understood in multi-index notation. We define a bilinear pairing between the spaces $S_{\kappa, \rho_{L}}$ and $H_{k, \bar{\rho}_{L}}$ by putting

$$
\{g, f\}=\left(g, \xi_{k}(f)\right)_{P e t}
$$

for $g \in S_{\kappa, \rho_{L}}$ and $f \in H_{k, \bar{\rho}_{L}}$. The pairing vanishes when $f$ is weakly holomorphic. Because of Proposition 4.3 the induced pairing between $S_{\kappa, \rho_{L}}$ and $H_{k, \bar{\rho}_{L}} / M_{k, \bar{\rho}_{L}}^{!}$is non-degenerate. So an $f \in H_{k, \bar{\rho}_{L}}$ is weakly holomorphic, if and only if $\{g, f\}=0$ for all $g \in S_{\kappa, \rho_{L}}$. 
Proposition 4.5. Let $g \in S_{\kappa, \rho_{L}}$ with Fourier expansion $g=\sum_{n, \nu} b(n, \nu) e(\operatorname{tr}(n \tau)) \chi_{\nu}$, and let

$$
f=\sum_{\mu \in L^{\prime} / L} \sum_{m \gg 0} c(m, \mu) f_{m, \mu}(\tau) \in H_{k, \bar{\rho}_{L}} .
$$

Then the pairing of $g$ and $f$ is equal to

$$
\{g, f\}=\sum_{\mu \in L^{\prime} / L} \sum_{m \gg 0} c(m, \mu) b(m, \mu) .
$$

Proof. This follows from (4.17) and (4.18) using the formula for the Petersson scalar product of Poincaré series with cusp forms, see e.g. [Luo, Section 2].

4.1. Whittaker forms and weak Maass forms. We end this section by explaining the relationship between Whittaker forms and weak Maass forms as defined in [BF]. Assume that $d=1$ so that $F=\mathbb{Q}$. Then there is no Koecher principle and there are nontrivial weak Maass forms. For simplicity we also assume that $k=k_{1}<0$.

A smooth function $f: \mathbb{H} \rightarrow S_{L}$ is called a harmonic weak Maass form (of weight $k$ with respect to $\tilde{\Gamma}$ and $\bar{\rho}_{L}$ ) if it satisfies:

(i) $\left.f\right|_{k, \bar{\rho}_{L}} \gamma=f$ for all $\gamma \in \tilde{\Gamma}$,

(ii) $\Delta_{k} f=0$, where $\Delta_{k}$ is the weight $k$ Laplacian,

(iii) there is a $S_{L}$-valued Fourier polynomial

$$
P_{f}(\tau)=\sum_{\mu \in L^{\prime} / L} \sum_{m \geq 0} c^{+}(m, \mu) q^{-m} \chi_{\mu}
$$

such that $f(\tau)-P_{f}(\tau)=O\left(e^{-\varepsilon v}\right)$ as $v \rightarrow \infty$ for some $\varepsilon>0$.

The Fourier polynomial $P_{f}$ is called the principal part of $f$. Here we denote the vector space of these harmonic weak Maass forms by $\mathcal{H}_{k, \bar{\rho}_{L}}$. Any weakly holomorphic modular form is a harmonic weak Maass form. We denote the subspace of weakly holomorphic modular forms by $\mathcal{M}_{k, \bar{\rho}_{L}}^{!}$.

Proposition 4.6. If $f \in H_{k, \bar{\rho}_{L}}$ is a harmonic Whittaker form, then

$$
\eta(f)=\left.\sum_{\gamma \in \tilde{\Gamma}_{\infty} \backslash \tilde{\Gamma}} f\right|_{k, \bar{\rho}_{L}} \gamma
$$

converges and defines an element of $\mathcal{H}_{k, \bar{\rho}_{L}}$. The map $\eta: H_{k, \bar{\rho}_{L}} \rightarrow \mathcal{H}_{k, \bar{\rho}_{L}}$ defined by $f \mapsto \eta(f)$ is an isomorphism. Its inverse is given by mapping a harmonic weak Maass form $g$ with principal part $P_{g}=\sum_{\mu} \sum_{m \geq 0} c^{+}(m, \mu) q^{-m} \chi_{\mu}$ to the harmonic Whittaker form

$$
f=\sum_{\mu} \sum_{m>0} c^{+}(m, \mu) f_{m, \mu}(\tau)
$$

The restriction of $\eta$ induces an isomorphism $M_{k, \bar{\rho}_{L}}^{!} \rightarrow \mathcal{M}_{k, \bar{\rho}_{L}}^{!}$.

The proof of the proposition follows from well known properties of non-holomorphic Poincaré series, see e.g. [He] or $[\mathrm{Br}]$, Chapter 1. Moreover, the operator $\xi_{k}$ on harmonic Whittaker forms is compatible with the corresponding operator on harmonic weak Maass forms of $[\mathrm{BF}]$. 


\section{THE THETA LIFT}

Here we define the regularized theta lift of Whittaker forms with parameter $s$. The images of the lift are automorphic Green functions for special divisors. We first do this when $\Re(s)$ is sufficiently large. For the general case we use meromorphic continuation in $s$, which can be obtained by means of spectral theory for $X_{K}$.

We use the setup of the previous sections. Throughout we assume that the compact open subgroup $K \subset H(\hat{\mathbb{Q}})$ fixes the lattice $L \subset V$ and acts trivially on $L^{\prime} / L$. Let $\Delta_{\mathbb{D}}$ be the Laplace operator induced by the the Casimir element of the Lie algebra of $\mathrm{SO}\left(V_{\sigma_{1}}\right)$ (or by the invariant metric on $\mathbb{D})$. We normalize it as in $[\mathrm{Br}](4.1)$.

Let $f$ be a Whittaker form of weight

$$
k=\left(\frac{2-n}{2}, \frac{2+n}{2}, \ldots, \frac{2+n}{2}\right)
$$

with parameter $s$ for $\tilde{\Gamma}$ and $\bar{\rho}_{L}$ (see Definition 4.1). We assume that $s>s_{0}:=1-k_{1}=n / 2$. Recall from Theorem 3.2 that the Siegel theta function $\Theta_{S}(\tau, z, h)$ associated to the lattice $L$ has weight $\left(\frac{n-2}{2}, \frac{2+n}{2}, \ldots, \frac{2+n}{2}\right)$ and representation $\rho_{L}$. Consequently, the pairing

$$
\left\langle f(\tau), \Theta_{S}(\tau, z, h)\right\rangle\left(v_{2} \cdots v_{d}\right)^{\ell / 2}
$$

is a scalar valued $\tilde{\Gamma}_{\infty}$-invariant function in $\tau$. We want to consider the theta integral

$$
\Phi(z, h, f)=\frac{1}{\sqrt{D}} \int_{\tilde{\Gamma}_{\infty} \backslash \mathbb{H}^{d}}\left\langle f(\tau), \Theta_{S}(\tau, z, h)\right\rangle\left(v_{2} \cdots v_{d}\right)^{\ell / 2} d \mu(\tau) .
$$

Because of the exponential growth of $f$, the integral does not converge. Similarly as in [Bo1] and [Br], we regularize it by prescribing the order of integration, namely we first integrate over $u$ and then over $v$. In the notation the regularization is indicated by the superscript "reg" at the integral.

Definition 5.1. The regularized theta lift of $f$ is defined as

$$
\begin{aligned}
\Phi(z, h, f) & =\frac{1}{\sqrt{D}} \int_{\tilde{\Gamma}_{\infty} \backslash \mathbb{H}^{d}}^{r e g}\left\langle f(\tau), \Theta_{S}(\tau, z, h)\right\rangle\left(v_{2} \cdots v_{d}\right)^{\ell / 2} d \mu(\tau) \\
& =\frac{1}{\sqrt{D}} \int_{v \in\left(\mathbb{R}_{>0}\right)^{d}}\left(\int_{u \in \mathcal{O}_{F} \backslash \mathbb{R}^{d}}\left\langle f(\tau), \Theta_{S}(\tau, z, h)\right\rangle d u\right)\left(v_{2} \cdots v_{d}\right)^{\ell / 2} \frac{d v}{\mathrm{~N}(v)^{2}} .
\end{aligned}
$$

Theorem 5.2. Let $f$ be a Whittaker form of parameter $s$ as above. If $\Re(s)>s_{0}+2$, then the regularized theta integral converges for $(z, h)$ outside a subset of $X_{K}$ of measure zero. It defines an integrable function on $X_{K}$.

To prove this Theorem, it suffices to consider for any $\mu \in L^{\prime} / L$ and any totally positive $m \in \partial_{F}^{-1}+Q(\mu)$ the theta integral

$$
\Phi_{m, \mu}(z, h, s):=\Phi\left(z, h, f_{m, \mu}(\cdot, s)\right)
$$

of the special Whittaker forms $f_{m, \mu}(\tau, s)$, see (4.13). We call $\Phi_{m, \mu}(z, h, s)$ the automorphic Green function of the divisor $Z(m, \mu)$. Later we will show that its regularized value at $s_{0}$ 
gives rise to a subharmonic Arakelov Green function for $Z(m, \mu)$ in the sense of [SABK]. Theorem 5.2 will follow from Theorem 5.3 below.

Let $F(a, b, c ; z)$ denote the Gauss hypergeometric function

$$
F(a, b, c ; z)=\sum_{n=0}^{\infty} \frac{(a)_{n}(b)_{n}}{(c)_{n}} \frac{z^{n}}{n !}
$$

see e.g. AbSt Chap. 15 or Er1 Vol. I Chap. 2. The circle of convergence of the series (5.3) is the unit circle $|z|=1$. For $\lambda \in V(\mathbb{R}), z \in \mathbb{D}$, and $s \in \mathbb{C}$ we put

$$
\phi(\lambda, z, s):=\frac{\Gamma\left(\frac{s}{2}+\frac{n}{4}\right)}{\Gamma(s+1)}\left(\frac{Q\left(\lambda_{1}\right)}{Q\left(\lambda_{1 z^{\perp}}\right)}\right)^{\frac{s}{2}+\frac{n}{4}} F\left(\frac{s}{2}+\frac{n}{4}, \frac{s}{2}-\frac{n}{4}+1, s+1 ; \frac{Q\left(\lambda_{1}\right)}{Q\left(\lambda_{1 z^{\perp}}\right)}\right) .
$$

This function has the invariance property $\phi(\lambda, z, s)=\phi(h \lambda, h z, s)$ for $h \in H(\mathbb{R})$. Notice that it is closely related to the secondary spherical function $\phi_{s}^{(2)}(h)$ on $\mathrm{SO}(n, 2)$ considered in [OT]. More precisely, for a totally positive $\lambda \in V$, we fix a base point $z_{0} \in \mathbb{D}$ such that $z_{0} \perp \lambda$. As in the proof of Theorem 4.7 of [BK] it is easily verified that

$$
\phi\left(\lambda, h z_{0}, s\right)=\frac{-2}{\Gamma\left(\frac{s}{2}-\frac{s_{0}}{2}+1\right)} \phi_{s}^{(2)}(h)
$$

for $h \in \mathrm{SO}\left(V_{\sigma_{1}}\right) \cong \mathrm{SO}(n, 2)$. It follows from [OT], Proposition 2.4.2, that $\phi(\lambda, z, s)$ satisfies the differential equation

$$
\Delta_{\mathbb{D}} \phi(\lambda, z, s)=\frac{1}{8}\left(s^{2}-s_{0}^{2}\right) \phi(\lambda, z, s)
$$

Theorem 5.3. Let $\mu \in L^{\prime} / L, m \in \partial_{F}^{-1}+Q(\mu)$, and $m \gg 0$. If $\Re(s)>s_{0}+2$, the regularized theta integral of $f_{m, \mu}(\tau, s)$ converges and is equal to

$$
\Phi_{m, \mu}(z, h, s)=\sum_{\substack{\lambda \in h(\mu+L) \\ Q(\lambda)=m}} \phi(\lambda, z, s) .
$$

The sum converges for $\Re(s)>s_{0}$ and $(z, h)$ outside a subset of $X_{K}$ of measure 0 . It defines an integrable function on $X_{K}$.

Proof. We first compute the theta integral $\Phi_{m, \mu}(z, h, s)$ formally. Afterwards we show the convergence of the infinite series representation in the statement of the theorem. The interchange of integration and summation in the computation of the theta integral is then justified a posteriori by the theorem of monotone convergence.

Inserting the definitions and carrying out the integration over $u$, we obtain

$$
\begin{aligned}
\Phi_{m, \mu}(z, h, s)= & C(m, k, s) \\
& \times \int_{\left(\mathbb{R}_{>0}\right)^{d}} \sum_{\substack{\lambda \in h(\mu+L) \\
Q(\lambda)=m}} \mathcal{M}_{s}\left(-4 \pi m_{1} v_{1}\right) e^{-4 \pi Q\left(\lambda_{1 z^{\perp}}\right) v_{1}} v_{1}^{-1}\left(v_{2} \cdots v_{d}\right)^{\ell / 2-2} d v .
\end{aligned}
$$

Here we have also used that $\operatorname{vol}\left(\mathcal{O}_{F} \backslash \mathbb{R}^{d}\right)=\sqrt{D}$. In view of Proposition 3.3 and (4.6), the integral converges for $\Re(s)>s_{0}+2$ and $(z, h) \in X_{K} \backslash Z(m, \mu)$. 
We put in the definition of $\mathcal{M}_{s}$ and interchange integration and summation. We find

$$
\begin{aligned}
\Phi_{m, \mu}(z, h, s)=C(m, k, s) & \sum_{\substack{\lambda \in h(\mu+L) \\
Q(\lambda)=m}} \int_{0}^{\infty} \frac{M_{-k_{1} / 2, s / 2}\left(4 \pi m_{1} v_{1}\right)}{\left(4 \pi m_{1} v_{1}\right)^{k_{1} / 2}} e^{-2 \pi m_{1} v_{1}+4 \pi Q\left(\lambda_{1 z}\right) v_{1}} \frac{d v_{1}}{v_{1}} \\
& \times \int_{0}^{\infty} e^{-4 \pi m_{2} v_{2}} v_{2}^{k_{2}-1} \frac{d v_{2}}{v_{2}} \cdots \int_{0}^{\infty} e^{-4 \pi m_{d} v_{d}} v_{d}^{k_{d}-1} \frac{d v_{d}}{v_{d}} .
\end{aligned}
$$

Inserting the value of $C(m, k, s)$ and carrying out the integration over $v_{2}, \ldots, v_{d}$, we obtain

$$
\begin{aligned}
\Phi_{m, \mu}(z, h, s)= & \frac{1}{\Gamma(s+1)\left(4 \pi m_{1}\right)^{k_{1} / 2}} \\
& \times \sum_{\substack{\lambda \in h(\mu+L) \\
Q(\lambda)=m}}^{\infty} \int_{0}^{\infty} M_{-k_{1} / 2, s / 2}\left(4 \pi m_{1} v_{1}\right) e^{-2 \pi m_{1} v_{1}+4 \pi Q\left(\lambda_{1 z}\right) v_{1}} v_{1}^{-k_{1} / 2} \frac{d v_{1}}{v_{1}} .
\end{aligned}
$$

The latter integral is a Laplace transform. It is equal to

$$
\frac{\left(4 \pi m_{1}\right)^{s / 2+1 / 2} \Gamma\left(\frac{s}{2}+\frac{n}{4}\right)}{\left(4 \pi Q\left(\lambda_{1 z^{\perp}}\right)\right)^{s / 2+n / 4}} F\left(\frac{s}{2}+\frac{n}{4}, \frac{s}{2}-\frac{n}{4}+1, s+1 ; \frac{m_{1}}{Q\left(\lambda_{1 z^{\perp}}\right)}\right),
$$

see e.g. [Er2] p. 215 (11). Consequently,

$$
\begin{aligned}
\Phi_{m, \mu}(z, h, s)= & \frac{\Gamma\left(\frac{s}{2}+\frac{n}{4}\right)}{\Gamma(s+1)} \\
& \times \sum_{\substack{\lambda \in h(\mu+L) \\
Q(\lambda)=m}}\left(\frac{m_{1}}{Q\left(\lambda_{1 z^{\perp}}\right)}\right)^{\frac{s}{2}+\frac{n}{4}} F\left(\frac{s}{2}+\frac{n}{4}, \frac{s}{2}-\frac{n}{4}+1, s+1 ; \frac{m_{1}}{Q\left(\lambda_{1 z^{\perp}}\right)}\right) .
\end{aligned}
$$

We now prove that the sum converges for $(z, h)$ outside a subset of measure zero to an integrable function on $X_{K}$. By reduction theory, the arithmetic group $\Gamma_{K}=H(\mathbb{Q}) \cap K$ acts with finitely many orbits on the set of $\lambda \in \mu+L$ with $Q(\lambda)=m$. We consider for a fixed $\lambda \in \mu+L$ with $Q(\lambda)=m$ the sum

$$
S(z):=\sum_{\gamma \in \Gamma_{K, \lambda} \backslash \Gamma_{K}} \phi(\gamma \lambda, z, s),
$$

where $\Gamma_{K, \lambda}=H_{\lambda}(\mathbb{Q}) \cap K$ and $H_{\lambda}$ denotes the stabilizer of $\lambda$ in $H$. It suffices to show that $S(z)$ converges outside a subset of measure zero and that $\int_{\Gamma_{K} \backslash \mathbb{D}} S(z) d \mu(z)<\infty$. According to Fubini's theorem, we have

$$
\int_{\Gamma_{K} \backslash \mathbb{D}} S(z) d \mu(z)=\int_{\Gamma_{K, \lambda} \backslash \mathbb{D}} \phi(\lambda, z, s) d \mu(z),
$$

and the desired convergence statement follows if we show that the integral on the right hand side is finite. Fixing a base point $z_{0} \in \mathbb{D}$ with $z_{0} \perp \lambda$, we may realize $\mathbb{D}$ as the coset 
space of $H(\mathbb{R})$ modulo the maximal compact subgroup given by the stabilizer of $z_{0}$. Hence it suffices to show that

$$
\int_{\Gamma_{K, \lambda} \backslash H(\mathbb{R})} \phi\left(\lambda, h z_{0}, s\right) d h<\infty
$$

where $d h$ denotes a Haar measure on $H(\mathbb{R})$. The latter integral is equal to

$$
\begin{aligned}
\int_{\Gamma_{K, \lambda} \backslash H(\mathbb{R})} \phi\left(h^{-1} \lambda, z_{0}, s\right) d h & =\int_{h \in \Gamma_{K, \lambda} \backslash H_{\lambda}(\mathbb{R})} \int_{h^{\prime} \in H_{\lambda}(\mathbb{R}) \backslash H(\mathbb{R})} \phi\left(h^{\prime-1} h^{-1} \lambda, z_{0}, s\right) d h^{\prime} d h \\
& =\operatorname{vol}\left(\Gamma_{K, \lambda} \backslash H_{\lambda}(\mathbb{R})\right) \int_{H_{\lambda}(\mathbb{R}) \backslash H(\mathbb{R})} \phi\left(h^{\prime-1} \lambda, z_{0}, s\right) d h^{\prime} .
\end{aligned}
$$

The convergence of the latter integral for $\Re(s)>s_{0}$ is proved in [OT], Proposition 3.1.1 (see also [BK], Section 4.2, for a comparison of the different setups).

Next we consider the singularities of $\Phi_{m, \mu}(z, h, s)$.

Lemma 5.4. Let $m \in F$ be totally positive. i) For all $\lambda \in L^{\prime}$ with $Q(\lambda)=m$ we have $0<m_{1} \leq Q\left(\lambda_{1 z^{\perp}}\right)$, and

$$
\frac{m_{1}}{Q\left(\lambda_{1 z^{\perp}}\right)}=\frac{2 m_{1}}{m_{1}+\left(Q\left(\lambda_{1 z^{\perp}}\right)-Q\left(\lambda_{1 z}\right)\right)} .
$$

ii) For any $\varepsilon>0$ and any compact subset $C \subset \mathbb{D}$, there are only finitely many $\lambda \in L^{\prime}$ with $Q(\lambda)=m$ and $\varepsilon<m_{1} / Q\left(\lambda_{1 z^{\perp}}\right)$ for some $z \in C$.

Proof. This follows by a straightforward computation and the fact that $Q\left(\lambda_{1 z^{\perp}}\right)-Q\left(\lambda_{1 z}\right)$ is a positive definite quadratic form on $V_{\sigma_{1}}$.

Theorem 5.5. Let $\mu \in L^{\prime} / L, m \in \partial_{F}^{-1}+Q(\mu)$, and $m \gg 0$. The series (5.7) and all its partial derivatives converge normally for $\Re(s)>s_{0}$ and $(z, h) \in X_{K} \backslash Z(m, \mu)$. For any point $\left(z_{0}, h_{0}\right) \in \mathbb{D} \times H(\hat{\mathbb{Q}})$ there is a neighborhood $U$ such that the function

$$
\Phi_{m, \mu}(z, h, s)-\sum_{\begin{array}{r}
\lambda \in h_{0}(\mu+L) \\
Q(\lambda)=m \\
\lambda_{1} \perp z_{0}
\end{array}} \phi(\lambda, z, s)
$$

is $C^{\infty}$ on $U$. Here the latter sum is finite.

Proof. Replacing the lattice $L$ by $h_{0} L$, we may assume without loss of generality that $h_{0}=1$. The condition $\lambda_{1} \perp z_{0}$ means that $\lambda_{1}$ is contained in the $n$-dimensional positive definite subspace $z_{0}^{\perp}$ of $V_{\sigma_{1}}$. Since $V_{\sigma_{2}}, \ldots, V_{\sigma_{d}}$ are positive definite, there are only finitely many $\lambda$ in the coset $\mu+L$ satisfying the condition under the sum in (5.8).

Let $U^{\prime} \subset \mathbb{D}$ be a compact neighborhood of $z_{0}$. Let $S_{1}$ be the set of all $\lambda \in \mu+L$ with $Q(\lambda)=m$ and $m_{1} / Q\left(\lambda_{1 z^{\perp}}\right)<1 / 2$ for all $z \in U^{\prime}$. Let $S_{2}$ be the set of all $\lambda \in \mu+L$ with $Q(\lambda)=m$ and $m_{1} / Q\left(\lambda_{1 z^{\perp}}\right) \geq 1 / 2$ for some $z \in U^{\prime}$. Then we have for $h \in K \subset H(\hat{\mathbb{Q}})$ that

$$
\Phi_{m, \mu}(z, h, s)=\sum_{\substack{\lambda \in h(\mu+L) \\ Q(\lambda)=m}} \phi(\lambda, z, s)=\sum_{\lambda \in S_{1}} \phi(\lambda, z, s)+\sum_{\lambda \in S_{2}} \phi(\lambda, z, s) .
$$


According to Lemma 5.4, the sum over $S_{2}$ is finite. Moreover,

$$
\sum_{\lambda \in S_{2}} \phi(\lambda, z, s)-\sum_{\substack{\lambda \in \mu+L \\ Q(\lambda)=m \\ \lambda_{1} \perp z_{0}}} \phi(\lambda, z, s)
$$

is a smooth function in a small neighborhood $U$ of $\left(z_{0}, 1\right)$.

Hence it suffices to show that the sum over $S_{1}$ converges normally for $z \in U^{\prime}$. Using the power series expansion of the Gauss hypergeometric function, we see that

$$
\phi(\lambda, z, s) \ll Q\left(\lambda_{1 z^{\perp}}\right)^{-s / 2-n / 4}
$$

for all $\lambda \in S_{1}$ and all $z \in U^{\prime}$. The same bound (with different implied constants) holds for all iterated partial derivatives of $\phi(\lambda, z, s)$. Consequently, it suffices to show that

$$
\sum_{\substack{\lambda \in h(\mu+L) \\ Q(\lambda)=m}} Q\left(\lambda_{1 z^{\perp}}\right)^{-s / 2-n / 4}
$$

converges normally on $U$. This can be proved by comparing with an integral as in the proof of Theorem 5.3 .

Since the Green function $\Phi_{m, \mu}(z, h, s)$ belongs to $L^{1}\left(X_{K}\right)$, we may view it as a current $\left[\Phi_{m, \mu, s}\right]$, that is, as a functional on smooth top degree differential forms on $X_{K}$ with compact support. For a bounded $C^{\infty}$-function $\alpha$ on $X_{K}$, we write

$$
\left[\Phi_{m, \mu, s}\right](\alpha)=\int_{X_{K}} \Phi_{m, \mu}(z, h, s) \alpha(z) d \mu(z) .
$$

Applying the Laplace operator, we obtain another current $\Delta_{\mathbb{D}}\left[\Phi_{m, \mu, s}\right]$, given by

$$
\left(\Delta_{\mathbb{D}}\left[\Phi_{m, \mu, s}\right]\right)(\alpha)=\int_{X_{K}} \Phi_{m, \mu}(z, h, s)\left(\Delta_{\mathbb{D}} \alpha\right)(z) d \mu(z) .
$$

Moreover, for any divisor $Y$ on $X_{K}$, there is a Dirac current $\delta_{Y}$, given by

$$
\delta_{Y}(\alpha)=\int_{Y} \alpha(z) \Omega^{n-1}
$$

We define the degree of the divisor $Y$ by $\operatorname{deg}(Y)=\delta_{Y}(1)$, provided the integral converges.

Remark 5.6. The degree of the first Chern class in $\mathrm{CH}^{1}\left(X_{K}\right)$ of the line bundle of modular forms of weight $w$ is given by $\operatorname{deg} c_{1}\left(\mathcal{M}_{w}\right)=w \operatorname{vol}\left(X_{K}\right)$.

Proof. The Petersson metric defines a hermitean metric on the line bundle of modular forms of weight $w$. Its first Chern form is $w \Omega$. The remark follows from the Poincaré-Lelong lemma.

Theorem 5.7. Let $\Re(s)>s_{0}$. The Green current $\left[\Phi_{m, \mu, s}\right]$ satisfies the following differential equation

$$
\Delta_{\mathbb{D}}\left[\Phi_{m, \mu, s}\right]=\frac{1}{8}\left(s^{2}-s_{0}^{2}\right)\left[\Phi_{m, \mu, s}\right]-\frac{n}{4 \Gamma\left(\frac{s}{2}-\frac{s_{0}}{2}+1\right)} \delta_{Z(m, \mu)} .
$$


Proof. In view of (5.5), the result follows from [OT], Theorem 3.2.1 (3) and Corollary 3.2.1. The comparison of the different normalizations of the Laplacian and the invariant measures is similar as in the proof of Theorem 4.7 of [BK].

5.1. The spectral expansion. For $d=1$ (when $F=\mathbb{Q}$ ) a meromorphic continuation of the Green function $\Phi_{m, \mu}(z, h, s)$ is obtained in $[\mathrm{Br}]$, employing Poincaré series built out of the functions $f_{m, \mu}(\tau, s)$ similarly as in Proposition 4.6. Since the corresponding Poincaré series do not converge when $d>1$, we cannot argue this way. Instead, we use the approach of [OT] $\S 6$ and [MW] $\S 4$ to prove meromorphic continuation in the sense of distributions by means of spectral theory. Moreover, we refine the argument to obtain a meromorphic continuation as a smooth function on $X_{K} \backslash Z(m, \mu)$.

We begin by computing the spectral expansion of $\Phi_{m, \mu}(z, h, s)$. Throughout this subsection we assume that $X_{K}$ is compact such that the Laplace operator has a discrete spectrum. This is always the case when $d>1$.

Recall that the Laplace operator $-\Delta_{\mathbb{D}}$ gives rise to a densely defined self-adjoint operator on $L^{2}\left(X_{K}\right)$ which is positive. Let $\Lambda \subset \mathbb{R}_{\geq 0}$ be the set of eigenvalues of $-\Delta_{\mathbb{D}}$. It is a countable set with no accumulation points. So we may write $\Lambda=\left\{\lambda_{k} ; k \in \mathbb{Z}_{\geq 0}\right\}$ with

$$
\lambda_{0} \leq \lambda_{1} \leq \lambda_{2} \leq \ldots
$$

where every $\lambda \in \mathbb{R}$ occurs with multiplicity $d(\lambda)$ given by the dimension of the corresponding eigenspace. Let $\left\{\varphi_{k}\right\} \subset \mathbb{C}^{\infty}\left(X_{K}\right)$ be an orthonormal system of eigenfunctions such that $-\Delta_{\mathbb{D}} \varphi_{k}=\lambda_{k} \varphi_{k}$. For any $k$ we choose $\alpha_{k} \in \mathbb{C}$ such that

$$
\lambda_{k}=-\frac{1}{8}\left(\alpha_{k}^{2}-s_{0}^{2}\right) .
$$

Then $\alpha_{k} \in\left[-s_{0}, s_{0}\right] \cup i \mathbb{R}$. For the eigenvalue 0 , the multiplicity $d(0)$ is equal to the number of connected components of $X_{K}$. We choose the suitably normalized characteristic functions of the components of $X_{K}$ as an orthonormal basis of the eigenspace.

Any function $\varphi \in L^{2}\left(X_{K}\right)$ has a spectral decomposition

$$
\varphi=\sum_{k=0}^{\infty}\left(\varphi, \varphi_{k}\right) \varphi_{k}
$$

where $(\varphi, \psi)=\int_{X_{K}} \varphi(z) \overline{\psi(z)} d \mu(z)$ denotes the scalar product on $L^{2}\left(X_{K}\right)$. The series converges in the $L^{2}$-norm. We now compute this expansion for $\Phi_{m, \mu}(z, h, s)$.

Theorem 5.8. Assume that $X_{K}$ is compact. The Green function $\Phi_{m, \mu}(z, h, s)$ has the spectral expansion

$$
\Phi_{m, \mu}(z, h, s)=\frac{2 n}{\Gamma\left(\frac{s}{2}-\frac{s_{0}}{2}+1\right)} \sum_{k=0}^{\infty} \frac{\delta_{Z(m, \mu)}\left(\overline{\varphi_{k}}\right)}{s^{2}-\alpha_{k}^{2}} \cdot \varphi_{k}(z, h) .
$$

Proof. This is an immediate consequence of Theorem 5.7 and (5.9). 
Corollary 5.9. As a distribution on $X_{K}$, the Green function $\Phi_{m, \mu}(z, h, s)$ has a meromorphic continuation in $s$ to the whole complex plane. At $s=s_{0}$ it has a simple pole with residue

$$
A(m, \mu):=2 \frac{\operatorname{deg}(Z(m, \mu))}{\operatorname{vol}\left(X_{K}\right)} .
$$

Proof. Using the Bessel inequality, one sees that the spectral expansion of $\Phi_{m, \mu}(z, h, s)$ given in Theorem 5.8 converges locally uniformly for $s \in \mathbb{C}$ with $s \neq \pm \alpha_{k}$ to an element of $L^{2}\left(X_{K}\right)$. This proves the meromorphic continuation.

The singularity at $s=s_{0}$ comes from the terms in the spectral expansion with eigenvalue $\lambda_{k}=0$. The sum over the remaining terms is holomorphic at $s_{0}$. Hence, the singularity is given by

$$
\frac{2 n}{\Gamma\left(\frac{s}{2}-\frac{s_{0}}{2}+1\right)} \sum_{\substack{k \geq 0 \\ \lambda_{k}=0}} \frac{\delta_{Z(m, \mu)}\left(\overline{\varphi_{k}}\right)}{s^{2}-s_{0}^{2}} \cdot \varphi_{k} .
$$

The eigenfunctions $\varphi_{k}$ contributing to this sum are the characteristic functions of the components of $X_{K}$ multiplied by the normalizing factor $\left(d(0) / \operatorname{vol}\left(X_{K}\right)\right)^{1 / 2}$. It is easily seen that for such eigenfunctions $\delta_{Z(m, \mu)}\left(\overline{\varphi_{k}}\right)=\left(d(0) \operatorname{vol}\left(X_{K}\right)\right)^{-1 / 2} \delta_{Z(m, \mu)}(1)$. Hence the $\operatorname{sum}(5.11)$ is equal to

$$
\frac{2 n\left(s^{2}-s_{0}^{2}\right)^{-1}}{\Gamma\left(\frac{s}{2}-\frac{s_{0}}{2}+1\right)} \cdot \frac{\operatorname{deg}(Z(m, \mu))}{\operatorname{vol}\left(X_{K}\right)} .
$$

This function has a simple pole at $s=s_{0}$ with the claimed residue.

Remark 5.10. The spectral expansion also implies that $\Gamma\left(\frac{s}{2}-\frac{s_{0}}{2}+1\right) \Phi_{m, \mu}(z, h, s)$ is invariant under the substitution $s \mapsto-s$.

We now refine the above argument, to obtain a meromorphic continuation in $s$ of $\Phi_{m, \mu}(z, h, s)$ as a continuous function on $X_{K} \backslash Z(m, \mu)$. Then the distribution differential equation of Theorem 5.7 and the elliptic regularity theorem imply that $\Phi_{m, \mu}(z, h, s)$ is actually real analytic on $X_{K} \backslash Z(m, \mu)$. The following lemma is known, see e.g. [Shu], Proposition 10.2. We include it here for completeness.

Lemma 5.11. Assume the notation of the beginning of this subsection.

(1) If $t>n$, then the series $\sum_{k \geq 0}\left(\lambda_{k}+1\right)^{-t}$ converges.

(2) For any integer $N>n$, there is a constant $C>0$ such that for all $k \geq 0$ we have

$$
\left\|\varphi_{k}\right\|_{\infty} \leq C\left(\lambda_{k}+1\right)^{N} \text {. }
$$

(3) If $\psi \in C^{\infty}\left(X_{K}\right)$, then for any $N \in \mathbb{Z}_{\geq 0}$ and for all $k \in \mathbb{Z}_{\geq 0}$ we have

$$
\left|\left(\psi, \varphi_{k}\right)\right| \leq\left(\lambda_{k}+1\right)^{-N}\left\|\left(-\Delta_{\mathbb{D}}+1\right)^{N} \psi\right\|_{2} .
$$

(4) If $\psi \in C^{\infty}\left(X_{K}\right)$, then the spectral expansion (5.9) converges uniformly towards $\psi$.

Proof. The first assertion is a consequence of Weyl's law which states that

$$
\#\left\{k ; \lambda_{k} \leq x\right\} \sim c x^{\delta / 2}, \quad x \rightarrow \infty,
$$


where $c>0$ is a constant and $\delta$ is the dimension (over $\mathbb{R}$ ) of the compact real Riemann manifold $X_{K}$.

The second assertion follows from the fact that for any integer $N>\delta / 2$ the pseudo differential operator $\left(-\Delta_{\mathbb{D}}+1\right)^{-N}$ has a continuous kernel function in $C\left(X_{K} \times X_{K}\right)$.

The third statement is an easy consequence of the self-adjointness of $-\Delta_{\mathbb{D}}$ and the Cauchy-Schwartz inequality. Finally, the last statement follows from (1), (2) and (3).

Theorem 5.12. For $(z, h) \in X_{K} \backslash Z(m, \mu)$, the Green function $\Phi_{m, \mu}(z, h, s)$ has a meromorphic continuation in $s$ to the whole complex plane. For fixed s outside the set of poles, the resulting function in $(z, h)$ is real analytic on $X_{K} \backslash Z(m, \mu)$.

Proof. Let $\sigma:[0,1] \rightarrow \mathbb{R}$ be a monotonous $C^{\infty}$-function such that $\sigma(t)=1$ for $t \leq 1 / 2$ and $\sigma(t)=0$ for $t \geq 3 / 4$. Besides the Poincaré series $\Phi_{m, \mu}(z, h, s)$ (see Theorem [5.3), we consider the Poincaré series

$$
\begin{aligned}
\tilde{\Phi}_{m, \mu}(z, h, s) & =\sum_{\substack{\lambda \in h(\mu+L) \\
Q(\lambda)=m}} \sigma\left(\frac{m_{1}}{Q\left(\lambda_{1 z^{\perp}}\right)}\right) \phi(\lambda, z, s), \\
F(z, h, s) & =\sum_{\substack{\lambda \in h(\mu+L) \\
Q(\lambda)=m}}\left[\Delta_{\mathbb{D}}\left(\sigma\left(\frac{m_{1}}{Q\left(\lambda_{1 z^{\perp}}\right)}\right) \phi(\lambda, z, s)\right)-\sigma\left(\frac{m_{1}}{Q\left(\lambda_{1 z^{\perp}}\right)}\right) \Delta_{\mathbb{D}}(\phi(\lambda, z, s))\right] .
\end{aligned}
$$

The difference of $\Phi_{m, \mu}(z, h, s)$ and $\tilde{\Phi}_{m, \mu}(z, h, s)$ is the Poincaré series

$$
\sum_{\substack{\lambda \in h(\mu+L) \\ Q(\lambda)=m}}\left(1-\sigma\left(\frac{m_{1}}{Q\left(\lambda_{1 z^{\perp}}\right)}\right)\right) \phi(\lambda, z, s) .
$$

As in the proof of Theorem 5.5 we see that it is locally finite, that is, for $(z, h)$ in any compact subset of $\mathbb{D} \times H(\hat{\mathbb{Q}})$, only finitely many terms are non-zero. Hence it defines a holomorphic function for all $s \in \mathbb{C}$, which is smooth for $(z, h) \in X_{K} \backslash Z(m, \mu)$. We now show that $\tilde{\Phi}_{m, \mu}(z, h, s)$ has a meromorphic continuation in $s$ which is continuous for $(z, h) \in X_{K}$. This implies the desired continuation of $\Phi_{m, \mu}(z, h, s)$.

As in the proof of Theorem 5.5 we see that $\tilde{\Phi}_{m, \mu}(z, h, s)$ converges normally for $\Re(s)>s_{0}$ and defines a smooth function on $X_{K}$. The Poincaré series $F(z, h, s)$ is locally finite and defines a smooth function, which is holomorphic in $s$ on the whole complex plane. Moreover, the differential equation (5.6) implies that

$$
\Delta_{\mathbb{D}} \tilde{\Phi}_{m, \mu}(z, h, s)=\frac{1}{8}\left(s^{2}-s_{0}^{2}\right) \tilde{\Phi}_{m, \mu}(z, h, s)+F(z, h, s)
$$

for $\Re(s)>s_{0}$. Hence the coefficients of the spectral expansion of $\tilde{\Phi}_{m, \mu}(z, h, s)$ are given by

$$
\left(\tilde{\Phi}_{m, \mu}(\cdot, s), \varphi_{k}\right)=\frac{8}{\alpha_{k}^{2}-s^{2}}\left(F(\cdot, s), \varphi_{k}\right),
$$

and we have

$$
\tilde{\Phi}_{m, \mu}(z, h, s)=\sum_{k=0}^{\infty} \frac{8}{\alpha_{k}^{2}-s^{2}}\left(F(\cdot, s), \varphi_{k}\right) \varphi_{k}(z, h) .
$$


Lemma 5.11 implies that the series converges locally uniformly for $s \in \mathbb{C}$ and $(z, h) \in X_{K}$. Consequently, it defines a meromorphic continuation in $s$ which is continuous in $(z, h)$.

Now the distribution differential equation of Theorem 5.7 and the elliptic regularity theorem imply that for fixed $s$ outside the set of poles, $\Phi_{m, \mu}(z, h, s)$ is actually real analytic for $(z, h) \in X_{K} \backslash Z(m, \mu)$.

5.2. Regularized Green functions. Here we define the regularized theta lift of a harmonic Whittaker form, that is, a Whittaker form with parameter $s_{0}$. We determine the singularities of the lift. In this subsection we do not have to assume that $X_{K}$ is compact. So we come back to the general setup of Section 2 .

Definition 5.13. Let $f \in H_{k, \bar{\rho}_{L}}$ and write

$$
f=\sum_{\mu \in L^{\prime} / L} \sum_{m \gg 0} c(m, \mu) f_{m, \mu}(\tau) .
$$

We define the regularized theta lift $\Phi(z, h, f)$ of $f$ to be the constant term in the Laurent expansion at $s=s_{0}$ of

$$
\Phi(z, h, s, f):=\sum_{\mu \in L^{\prime} / L} \sum_{m \gg 0} c(m, \mu) \Phi_{m, \mu}(z, h, s) .
$$

If $\mu \in L^{\prime} / L$ and $m \in \partial_{F}^{-1}+Q(\mu)$ is totally positive, we briefly write $\Phi_{m, \mu}(z, h)$ for the regularized theta lift of the harmonic Whittaker form $f_{m, \mu}(\tau)$, that is, for the constant term in the Laurent expansion of $\Phi_{m, \mu}(z, h, s)$ at $s=s_{0}$.

For a harmonic Whittaker form $f \in H_{k, \bar{\rho}_{L}}$ as in (5.13) we define a divisor $Z(f) \in$ $\operatorname{Div}\left(X_{K}\right)_{\mathbb{C}}$ by

$$
Z(f)=\sum_{\mu \in L^{\prime} / L} \sum_{m \gg 0} c(m, \mu) Z(m, \mu) .
$$

Moreover, by means of the quantities $A(m, \mu)$ of Corollary 5.9 we define

$$
A(f)=\sum_{\mu \in L^{\prime} / L} \sum_{m \gg 0} c(m, \mu) A(m, \mu) .
$$

In view of Corollary 5.9 we have

$$
\Phi(z, h, f)=\lim _{s \rightarrow s_{0}}\left(\Phi(z, h, s, f)-\frac{A(f)}{s-s_{0}}\right) .
$$

If $Y$ is an irreducible Cartier divisor on a normal complex space $X$, we say that a real analytic function $F$ on $X \backslash Y$ has a logarithmic singularity along $Y$, if for any $x \in Y$ there is a neighborhood $U \subset X$ and a local equation $G$ for $Y$ such that $F-\log |G|$ can be continued to a real analytic function on $U$. We extend this definition $\mathbb{C}$-linearly to $\operatorname{Div}\left(X_{K}\right)_{\mathbb{C}}$.

Theorem 5.14. Assume that $n>0$. For $f \in H_{k, \bar{\rho}_{L}}$ the function $\Phi(z, h, f)$ is real analytic on $X_{K} \backslash Z(f)$. It has a logarithmic singularity along the divisor $-2 Z(f)$. 
Proof. It suffices to show that for $\mu \in L^{\prime} / L$ and totally positive $m \in \partial_{F}^{-1}+Q(\mu)$, the function $\Phi_{m, \mu}(z, h)$ is real analytic on $X_{K} \backslash Z(m, \mu)$ and has a logarithmic singularity along the divisor $-2 Z(m, \mu)$. To this end we show that for any point $\left(z_{0}, h_{0}\right) \in \mathbb{D} \times H(\hat{\mathbb{Q}})$ the function

$$
\Phi_{m, \mu}(z, h)+\sum_{\substack{\lambda \in h_{0}(\mu+L) \\ Q(\lambda)=m \\ \lambda_{1} \perp z_{0}}} \log \left|Q\left(\lambda_{1 z}\right)\right|
$$

is real analytic in a neighborhood of $\left(z_{0}, h_{0}\right)$.

Since the residue of $\Phi_{m, \mu}(z, h, s)$ at $s=s_{0}$ does not depend on $(z, h)$, the proof of Theorem 5.12 also shows that the function

$$
\Phi_{m, \mu}(z, h)-\sum_{\substack{\lambda \in h_{0}(\mu+L) \\ Q(\lambda)=m \\ \lambda_{1} \perp z_{0}}} \phi\left(\lambda, z, s_{0}\right)
$$

is real analytic in a neighborhood of $\left(z_{0}, h_{0}\right)$. Hence it suffices to show that

$$
\phi\left(\lambda, z, s_{0}\right)+\log \left|Q\left(\lambda_{1 z}\right)\right|
$$

extends to a real analytic function on $\mathbb{D}$. This follows from Lemma 5.15 below.

Lemma 5.15. If $n>0$, the function

$$
\frac{2}{n} w^{n / 2} F(n / 2,1, n / 2+1, w)+\log (1-w)
$$

extends to a real analytic function near $w=1$.

Proof. We use the integral representation

$$
\frac{2}{n} w^{n / 2} F(n / 2,1, n / 2+1, w)=\int_{0}^{1}(t w)^{n / 2}(1-t w)^{-1} \frac{d t}{t}=\int_{0}^{w} t^{n / 2}(1-t)^{-1} \frac{d t}{t},
$$

see for instance $\mathrm{AbSt}$ (15.3.1). Comparing this with $\log (1-w)=-\int_{0}^{w} \frac{d t}{1-t}$, we see that

$$
\frac{2}{n} w^{n / 2} F(n / 2,1, n / 2+1, w)+\log (1-w)=\int_{0}^{w} \frac{t^{n / 2-1}-1}{1-t} d t .
$$

If $n=1$ this is equal to $\int_{0}^{w} \frac{d t}{t+\sqrt{t}}$, if $n=2$ it vanishes identically, and if $n \geq 3$ it is equal to

$$
-\sum_{k=0}^{n-3} \int_{0}^{w} \frac{t^{k / 2}}{1+\sqrt{t}} d t
$$

In all cases, the resulting function is real analytic near $w=1$.

Corollary 5.16. The differential form $d d^{c} \Phi(f)$ extends to a smooth form on $X_{K}$, which is a (harmonic) Poincaré dual form for $Z(f)$. The current $[\Phi(f)]$ induced by $\Phi(z, h, f)$ satisfies the $d d^{c}$-equation

$$
d d^{c}[\Phi(f)]+\delta_{Z(f)}=\left[d d^{c} \Phi(f)\right]
$$


Proof. The corollary follows from Theorem 5.14 by means of the usual Poincaré-Lelong argument, see e.g. [SABK], Chapter II.1.4, Theorem 2.

Remark 5.17. The current $[\Phi(f)]$ induced by $\Phi(z, h, f)$ satisfies the differential equation

$$
\Delta_{\mathbb{D}}[\Phi(f)]+\frac{n}{4} \delta_{Z(f)}=\frac{n}{8}[A(f)] .
$$

Proof. This is a direct consequence of Theorem 5.7 and Corollary 5.9.

\section{THE THETA LIFT AND MEROMORPHIC MODULAR FORMS}

We continue to use the notation of the previous section. Here we investigate the relationship of the regularized theta lift and the Kudla-Millson lift (see [KM1], [KM2], [KM3]). We use the approach of [BF]. As an application we construct explicit meromorphic modular forms on $X_{K}$ whose divisors are supported on Heegner divisors. They are analogous to the automorphic products constructed by Borcherds [Bo1]. However, notice that there are no Fourier expansions and therefore no product expansions when $X_{K}$ is compact. Consequently, Borcherds' argument to prove important properties of the lift (such as e.g. meromorphicity) cannot be employed when $d>1$.

6.1. The relationship with a regularized Kudla-Millson lift. Recall that $\kappa$ is the dual weight for $k$ given by

$$
\kappa=\left(2-k_{1}, k_{2}, \ldots, k_{d}\right)=\left(\frac{n+2}{2}, \ldots, \frac{n+2}{2}\right) .
$$

Proposition 6.1. Let $\mu \in L^{\prime} / L$ and $m \in \partial_{F}^{-1}+Q(\mu)$ be totally positive. For $\Re(s)>s_{0}+2$ we have the identity

$$
d d^{c} \Phi_{m, \mu}(z, h, s)=\frac{1}{\sqrt{D}} \int_{\tilde{\Gamma}_{\infty} \backslash \mathbb{H}^{d}}^{r e g}\left\langle\overline{\delta_{k}\left(f_{m, \mu}(\tau, s)\right)}, \Theta_{K M}(\tau, z, h)\right\rangle v^{\kappa} d \mu(\tau) .
$$

Here $\delta_{k}$ is the differential operator defined in (4.2).

Proof. According to Proposition 3.1 we have

$$
d d^{c} \Theta_{S}(\tau, z, h)=-L_{\kappa}^{(1)} \Theta_{K M}(\tau, z, h) .
$$

Moreover, writing $\eta=\left(v_{2} \cdots v_{d}\right)^{\ell / 2} d \tau_{1} d \mu\left(\tau_{2}\right) \cdots d \mu\left(\tau_{d}\right)$, we have the identity of differential forms on $\mathbb{H}^{d}$ :

$$
-\left(L_{\kappa}^{(1)} \Theta_{K M}(\tau, z, h)\right)\left(v_{2} \cdots v_{d}\right)^{\ell / 2} d \mu(\tau)=\bar{\partial}\left(\Theta_{K M}(\tau, z, h) \eta\right) .
$$

In view of (4.6), when $\Re(s)$ is sufficiently large, we may interchange the regularized theta integral in the definition of $\Phi_{m, \mu}(z, h, s)$ with the operator $d d^{c}$. By means of the above 
identities we obtain

$$
\begin{aligned}
d d^{c} \Phi_{m, \mu}(z, h, s) & =\frac{1}{\sqrt{D}} \int_{\tilde{\Gamma}_{\infty} \backslash \mathbb{H}^{d}}^{r e g}\left\langle f_{m, \mu}(\tau, s), d d^{c} \Theta_{S}(\tau, z, h)\right\rangle\left(v_{2} \cdots v_{d}\right)^{\ell / 2} d \mu(\tau) \\
& =\frac{1}{\sqrt{D}} \int_{\tilde{\Gamma}_{\infty} \backslash \mathbb{H}^{d}}^{r e g}\left\langle f_{m, \mu}(\tau, s),-L_{\kappa}^{(1)} \Theta_{K M}(\tau, z, h)\right\rangle\left(v_{2} \cdots v_{d}\right)^{\ell / 2} d \mu(\tau) \\
& =\frac{1}{\sqrt{D}} \int_{\tilde{\Gamma}_{\infty} \backslash \mathbb{H}^{d}}^{r e g}\left\langle f_{m, \mu}(\tau, s), \bar{\partial} \Theta_{K M}(\tau, z, h) \eta\right\rangle .
\end{aligned}
$$

Using the product rule, we find

$$
\begin{aligned}
d d^{c} \Phi_{m, \mu}(z, h, s)= & \frac{1}{\sqrt{D}} \int_{\tilde{\Gamma}_{\infty} \backslash \mathbb{H}^{d}}^{r e g} d\left\langle f_{m, \mu}(\tau, s), \Theta_{K M}(\tau, z, h) \eta\right\rangle \\
& -\frac{1}{\sqrt{D}} \int_{\tilde{\Gamma}_{\infty} \backslash \mathbb{H}^{d}}^{r e g}\left\langle\bar{\partial}\left(f_{m, \mu}(\tau, s)\right), \Theta_{K M}(\tau, z, h) \eta\right\rangle .
\end{aligned}
$$

For the second summand on the right hand side we notice that

$$
\begin{aligned}
\bar{\partial}\left(f_{m, \mu}(\tau, s)\right) \eta & =-\left(L_{k}^{(1)} f_{m, \mu}(\tau, s)\right)\left(v_{2} \cdots v_{d}\right)^{\ell / 2} d \mu(\tau) \\
& =-\overline{\delta_{k}\left(f_{m, \mu}(\tau, s)\right)}\left(v_{1} \cdots v_{d}\right)^{\ell / 2} d \mu(\tau) .
\end{aligned}
$$

Hence this term gives the right hand side of the formula stated in the proposition.

Consequently, it suffices to prove that the first summand on the right hand side of (6.1) vanishes for $\Re(s)>s_{0}+2$. For $T>0$ we let $R_{T} \subset \mathbb{R}_{>0}^{d}$ be the rectangle

$$
R_{T}=[1 / T, T] \times \cdots \times[1 / T, T] .
$$

Using the invariance of the integrand under translations, we find by Stokes' theorem that

$$
\int_{\tilde{\Gamma}_{\infty} \backslash \mathbb{H}^{d}}^{r e g} d\left\langle f_{m, \mu}(\tau, s), \Theta_{K M}(\tau, z, h) \eta\right\rangle=\lim _{T \rightarrow \infty} \int_{\partial R_{T}} \int_{\mathcal{O}_{F} \backslash \mathbb{R}^{d}}\left\langle f_{m, \mu}(\tau, s), \Theta_{K M}(\tau, z, h) \eta\right\rangle .
$$

Inserting (3.4), (3.14), and (4.13), and carrying out the integration over $u$, we see that this is equal to

$$
\begin{aligned}
& \sqrt{D} C(m, k, s) \lim _{T \rightarrow \infty} \int_{\partial R_{T}} \mathcal{M}_{s}\left(-4 \pi m_{1} v_{1}\right) \\
& \quad \times \sum_{\substack{\lambda \in h(\mu+L) \\
Q(\lambda)=m}} P_{K M}\left(z, \sqrt{v_{1}} \lambda_{1}\right) e^{-4 \pi Q\left(\lambda_{1 z^{\perp}}\right) v_{1}}\left(v_{2} \cdots v_{d}\right)^{\ell / 2-2} d v_{2} \cdots d v_{d} .
\end{aligned}
$$

Only the parts of the boundary where $v_{1}=1 / T$ or $v_{1}=T$ give a non-zero contribution. Carrying out the integration over $v_{2}, \ldots, v_{d}$, we see that the $v_{1}=1 / T$ contribution is equal to a constant times

$$
\lim _{T \rightarrow \infty} T^{k_{1} / 2} M_{-k_{1} / 2, s / 2}\left(4 \pi m_{1} / T\right) \sum_{\substack{\lambda \in h(\mu+L) \\ Q(\lambda)=m}} P_{K M}\left(z, T^{-1 / 2} \lambda_{1}\right) e^{-2 \pi\left(Q\left(\lambda_{1 z} \perp\right)-Q\left(\lambda_{1 z}\right)\right) / T} .
$$


The later sum is up to a constant factor equal to the $m$-th Fourier coefficient of

$$
\Theta_{K M}(u+i(1 / T, 1, \ldots, 1), z, h) .
$$

Hence it converges and satisfies the growth estimate of Proposition 3.4 as $1 / T \rightarrow 0$. So the asymptotic behavior of the $M$-Whittaker function (4.6) implies that the limit vanishes for $\Re(s)>s_{0}+2$. On the other hand, the $v_{1}=T$ contribution is easily seen to vanish for $(z, h) \in X_{K} \backslash Z(m, \mu)$. This proves the proposition.

6.2. Eisenstein series and theta integrals. The right hand side of the formula of Proposition 6.1 converges for $\Re(s)>s_{0}+2$. It has a meromorphic continuation to the whole complex plane, since the left hand side has. It is actually holomorphic at $s=s_{0}$. We now modify the integral representation on the right hand side in order to obtain an expression which converges near $s_{0}$. This is done by subtracting the "Eisenstein contribution" of $\Theta_{K M}(\tau, z, h)$. The remaining "cuspidal contribution" satisfies a better growth estimate as $v_{i} \rightarrow 0$ and therefore leads to a larger domain of convergence.

We briefly summarize some facts on the Siegel-Weil formula, see e.g. [KR], [Ku3] for more details. Let $\chi_{V}$ denote the quadratic character of $\mathbb{A}_{F}^{\times} / F^{\times}$associated to $V$ given by

$$
\chi_{V}(x)=\left(x,(-1)^{\ell(\ell-1) / 2} \operatorname{det}(V)\right)_{F} .
$$

Here $\operatorname{det}(V)$ denotes the Gram determinant of $V$ and $(\cdot, \cdot)_{F}$ is the Hilbert symbol of $F$. Let $P \subset G$ be the parabolic subgroup of upper triangular matrices. For $s \in \mathbb{C}$ and a standard section $\Phi(s)$ of the principal series representation $I\left(s, \chi_{V}\right)$ induced by $\chi_{V}|\cdot|^{s}$, we have the Eisenstein series

$$
E(g, s, \Phi)=\sum_{\gamma \in P(F) \backslash G(F)} \Phi(\gamma g) .
$$

It converges for $\Re(s)>1$ and has a meromorphic continuation to the whole complex plane.

Recall that if $v=\sigma_{j}$ is an infinite prime, then the corresponding local induced representation $I\left(s, \chi_{V, \sigma_{j}}\right)$ is generated by the sections

$$
\Phi_{\mathbb{R}}^{l_{j}}\left(k_{\alpha}, \phi\right)=\chi_{1 / 2}\left(k_{\alpha}, \phi\right)^{2 l_{j}}= \pm e^{i l_{j} \alpha}
$$

for $l_{j} \in \frac{1}{2} \mathbb{Z}$ satisfying $l_{j} \equiv \ell / 2(\bmod \mathbb{Z})$. Here $\left(k_{\alpha}, \phi\right) \in \widetilde{\mathrm{SO}}_{2}(\mathbb{R})$ is given by (3.1) and $\chi_{1 / 2}$ is the character defined in (3.2). If $l=\left(l_{1}, \ldots, l_{d}\right)$ is a $d$-tuple of such half-integers we put $\Phi_{\infty}^{l}=\prod_{j} \Phi_{\mathbb{R}}^{l_{j}}$. If $\Phi_{f}(s)$ is a standard section of the non-archimedian induced representation, we obtain an Eisenstein series of weight $l$ on $\mathbb{H}^{d}$ by putting

$$
E\left(\tau, s, l ; \Phi_{f}\right)=v^{-l / 2} E\left(\tilde{g}_{\tau}, s, \Phi_{f} \otimes \Phi_{\infty}^{l}\right),
$$

where $\tilde{g}_{\tau} \in \tilde{G}_{\mathbb{R}}$ with the property that $\tilde{g}_{\tau}(i, \ldots, i)=\tau$.

The Weil representation gives rise to a $\tilde{G}_{\mathbb{A}}$-intertwining map

$$
\lambda: S\left(V\left(\mathbb{A}_{F}\right)\right) \longrightarrow I\left(s_{0}, \chi_{V}\right), \quad \lambda(\varphi)(g)=(\omega(g) \varphi)(0),
$$

where $s_{0}=\ell / 2-1=n / 2$. We also write $\lambda(\varphi)$ for the unique standard section of $I\left(s, \chi_{V}\right)$ whose value at $s_{0}$ is equal to $\lambda(\varphi)$. The map $\lambda$ factors into $\lambda=\lambda_{\infty} \otimes \lambda_{f}$, where $\lambda_{\infty}$ and $\lambda_{f}$ 
are the analogous intertwining maps at the finite and the infinite places, respectively. We obtain a vector valued Eisenstein series for $\tilde{\Gamma}$ of weight $l$ with representation $\rho_{L}$ by putting

$$
E_{L}(\tau, s, l)=\sum_{\mu \in L^{\prime} / L} E\left(\tau, s, l ; \lambda_{f}\left(\chi_{\mu}\right)\right) \chi_{\mu} .
$$

Note that if the class number of $F$ is one, we have that

$$
E_{L}(\tau, s, l)=\left.\sum_{\gamma \in \tilde{\Gamma}_{\infty} \backslash \tilde{\Gamma}}\left(v_{1}^{\left(s+1-l_{1}\right) / 2} \cdots v_{d}^{\left(s+1-l_{d}\right) / 2} \chi_{0}\right)\right|_{l, \rho_{L}} \gamma .
$$

In general, it is a finite sum over such Eisenstein series. We will be interested in the special value $E_{L}(\tau, \kappa):=E_{L}\left(\tau, s_{0}, \kappa\right)$ at $s_{0}$.

For the rest of this section we assume that $V$ is anisotropic over $F$ or that its Witt rank is smaller than $n$. Note that this condition is automatically fulfilled when $d>1$ or $n>2$. Employing the Siegel-Weil formula (see e.g. [KR] and We2]), it can be shown that the average value of the Kudla-Millson theta function on $X_{K}$ is the given by an Eisenstein series of weight $\kappa$. More precisely, we have

$$
E_{L}(\tau, \kappa)=-\frac{1}{\operatorname{vol}\left(X_{K}\right)} \int_{X_{K}} \Theta_{K M}(\tau, z, h) \Omega^{n-1} .
$$

Kudla proved this for $F=\mathbb{Q}$ in $[\mathrm{Ku} 3$, Corollary 4.16], and the argument for general $F$ is analogous. Moreover, as in [Ku3, Remark 2.8], it can be proved that $E_{L}(\tau, \kappa)$ is holomorphic in $\tau$ and therefore defines an element of $M_{\kappa, \rho_{L}}$.

At the cusp $\infty$, the Eisenstein series has a Fourier expansion of the form

$$
E_{L}(\tau, \kappa)=\chi_{0}+\sum_{\mu \in L^{\prime} / L} \sum_{m>0} B(m, \mu) e(\operatorname{tr}(m \tau)) \chi_{\mu} .
$$

The coefficients $B(m, \mu)$ can be computed explicitly using the argument of [KY], [Scho], or $[\mathrm{BK}$. However, we will not need that.

The differential form $-E_{L}(\tau, \kappa) \Omega$ can be viewed as the average of $\Theta_{K M}(\tau, z, h)$. We define the cuspidal part of the Kudla-Millson theta function by

$$
\tilde{\Theta}_{K M}(\tau, z, h)=\Theta_{K M}(\tau, z, h)+E_{L}(\tau, \kappa) \Omega .
$$

It is rapidly decreasing at all cusps of $\tilde{\Gamma}$.

Proposition 6.2. Assume the above hypothesis on $V$.

(i) The function $\tilde{\Theta}_{K M}(\tau, z, h) v^{\kappa / 2}$ is bounded on $\mathbb{H}^{d}$.

(ii) For $v_{i} \rightarrow 0$ we have uniformly in $u$ that $\tilde{\Theta}_{K M}(\tau, z, h)=O\left(v^{-\kappa / 2}\right)$.

(iii) For $m \in \partial_{F}^{-1}$ the $m$-th Fourier coefficient of $\tilde{\Theta}_{K M}(\tau, z, h)$ is bounded by $O\left(v_{1}^{-\kappa_{1} / 2}\right)$ as $v_{i} \rightarrow 0$.

Proof. Since $\tilde{\Theta}_{K M}(\tau, z, h)$ is rapidly decreasing, (i) and (ii) follow by the usual argument. It remains to prove (iii). The behavior of the Fourier coefficients as $v_{1} \rightarrow 0$ is a direct consequence of (ii). Moreover, since $\tilde{\Theta}_{K M}(\tau, z, h)$ is holomorphic in $\tau_{2}, \ldots, \tau_{d}$ its Fourier coefficients are bounded as $v_{i} \rightarrow 0$ for $i=2, \ldots, d$. 
Proposition 6.3. We have

$$
d d^{c} \Phi_{m, \mu}(z, h, s)=\frac{1}{\sqrt{D}} \int_{\tilde{\Gamma}_{\infty} \backslash \mathbb{H}^{d}}^{r e g}\left\langle\overline{\delta_{k}\left(f_{m, \mu}(\tau, s)\right)}, \tilde{\Theta}_{K M}(\tau, z, h)\right\rangle v^{\kappa} d \mu(\tau)-\frac{B(m, \mu) \Omega}{\Gamma\left(\frac{s}{2}-\frac{s_{0}}{2}+1\right)} .
$$

Here the regularized integral converges locally uniformly for $\Re(s)>1$.

Proof. According to Proposition 6.1 we have

$$
\begin{aligned}
d d^{c} \Phi_{m, \mu}(z, h, s)= & \frac{1}{\sqrt{D}} \int_{\tilde{\Gamma}_{\infty} \backslash \mathbb{H}^{d}}^{r e g}\left\langle\overline{\delta_{k}\left(f_{m, \mu}(\tau, s)\right)}, \tilde{\Theta}_{K M}(\tau, z, h)\right\rangle v^{\kappa} d \mu(\tau) \\
& -\frac{1}{\sqrt{D}} \int_{\tilde{\Gamma}_{\infty} \backslash \mathbb{H}^{d}}^{r e g}\left\langle\overline{\delta_{k}\left(f_{m, \mu}(\tau, s)\right)}, E_{L}(\tau, \kappa) \Omega\right\rangle v^{\kappa} d \mu(\tau) .
\end{aligned}
$$

We have to compute the latter integral. A direct computation shows that

$$
\begin{aligned}
\overline{\delta_{k}\left(f_{m, \mu}(\tau, s)\right)}= & C(m, k, s)\left(s+s_{0}\right)\left(4 \pi m_{1}\right)^{-k_{1} / 2} \\
& \times v_{1}^{k_{1} / 2-1} M_{1-k_{1} / 2, s / 2}\left(4 \pi m_{1} v_{1}\right) e^{-2 \pi m_{2} v_{2}} \cdots e^{-2 \pi m_{d} v_{d}} e(-\operatorname{tr}(m u)) \chi_{\mu} .
\end{aligned}
$$

Inserting this and carrying out the integrations over $u$ and $v_{2}, \ldots, v_{d}$, we see that the second integral on the right hand side of (6.8) is equal to

$$
B(m, \mu) \Omega \cdot \frac{s+s_{0}}{\Gamma(s+1)} \int_{0}^{\infty}\left(4 \pi m_{1} v_{1}\right)^{-k_{1} / 2} M_{1-k_{1} / 2, s / 2}\left(4 \pi m_{1} v_{1}\right) e^{-2 \pi m_{1} v_{1}} \frac{d v_{1}}{v_{1}} .
$$

This is a Laplace transform, which can be computed by means of [Er2] p. 215 (11). We obtain for the second integral on the right hand side of (6.8)

$$
\frac{B(m, \mu) \Omega}{\Gamma\left(\frac{s}{2}-\frac{s_{0}}{2}+1\right)} \text {. }
$$

This proves the formula of the proposition.

We now prove the convergence statement for the integral. According to (6.9) and (4.6) we have

$$
\overline{\delta_{k}\left(f_{m, \mu}(\tau, s)\right)}=O\left(v_{1}^{\Re(s) / 2+\left(k_{1}-1\right) / 2}\right), \quad v_{i} \rightarrow 0 .
$$

By means of Proposition 6.2 (iii), we see that

$$
\int_{\mathcal{O}_{F} \backslash \mathbb{R}^{d}}\left\langle\overline{\delta_{k}\left(f_{m, \mu}(\tau, s)\right)}, \tilde{\Theta}_{K M}(\tau, z, h)\right\rangle v^{\kappa} d u=O\left(v_{1}^{\Re(s) / 2+1 / 2}\left(v_{2} \cdots v_{d}\right)^{n / 2+1}\right),
$$

as $v_{i} \rightarrow 0$. On the other hand, in view of (4.7), this quantity is bounded as $v_{i} \rightarrow \infty$. Consequently,

$$
\int_{v \in \mathbb{R}_{>0}^{d}}\left(\int_{\mathcal{O}_{F} \backslash \mathbb{R}^{d}}\left\langle\overline{\delta_{k}\left(f_{m, \mu}(\tau, s)\right)}, \tilde{\Theta}_{K M}(\tau, z, h)\right\rangle v^{\kappa} d u\right) \frac{d v}{\mathrm{~N}(v)^{2}}
$$

converges when $\Re(s)>1$. 
6.3. Regularized Green functions and the Kudla-Millson lift of cusp forms. For a cusp form $g \in S_{\kappa, \rho_{L}}$ we define the Kudla-Millson lift by

$$
\Lambda(z, h, g)=\left(\Theta_{K M}(\tau, z, h), g(\tau)\right)_{P e t} .
$$

The theta integral converges and defines a closed harmonic 2-form on $X_{K}$. The following theorem is a generalization of $[\mathrm{BF}]$ Theorem 6.1 to our situation.

Theorem 6.4. Let $f \in H_{k, \bar{\rho}_{L}}$. We write

$$
f=\sum_{\mu \in L^{\prime} / L} \sum_{m \gg 0} c(m, \mu) f_{m, \mu}(\tau)
$$

and define

$$
B(f)=\sum_{\mu \in L^{\prime} / L} \sum_{m \gg 0} c(m, \mu) B(m, \mu) .
$$

Then we have the identity

$$
d d^{c} \Phi(z, h, f)=\Lambda\left(z, h, \xi_{k}(f)\right)-B(f) \Omega .
$$

Proof. We first assume that $n>2$. Then it follows from Proposition 6.3 that the regularized Green function $\Phi_{m, \mu}(z, h)$ satisfies the identity

$$
d d^{c} \Phi_{m, \mu}(z, h)=\frac{1}{\sqrt{D}} \int_{\tilde{\Gamma}_{\infty} \backslash \mathbb{H}^{d}}\left\langle\overline{\delta_{k}\left(f_{m, \mu}(\tau)\right)}, \tilde{\Theta}_{K M}(\tau, z, h)\right\rangle v^{\kappa} d \mu(\tau)-B(m, \mu) \Omega
$$

Notice that the regularized theta integral in Proposition 6.3 converges near $s=s_{0}$ when $n>2$. Moreover, because of (4.17) and Proposition 6.2, the theta integral in (6.12) is well defined without any regularization! By the unfolding argument we see that it is equal to

$$
\int_{\tilde{\Gamma} \backslash \mathbb{H}^{d}}\left\langle\overline{\xi_{k}\left(f_{m, \mu}(\tau)\right)}, \tilde{\Theta}_{K M}(\tau, z, h)\right\rangle v^{\kappa} d \mu(\tau) .
$$

Since the integral of the cusp form $\xi_{k}\left(f_{m, \mu}\right)$ against the Eisenstein series $E_{L}(\tau, \kappa)$ vanishes, we obtain the assertion.

If $n \geq 1$, then one can show by means of Proposition 6.3 that $d d^{c} \Phi_{m, \mu}(z, h)$ is equal to the value at $s^{\prime}=0$ of the holomorphic continuation in $s^{\prime}$ of

$$
\frac{1}{\sqrt{D}} \int_{\tilde{\Gamma}_{\infty} \backslash \mathbb{H}^{d}}\left\langle\overline{\delta_{k}\left(f_{m, \mu}(\tau)\right)}, \tilde{\Theta}_{K M}(\tau, z, h)\right\rangle v^{\kappa} \mathrm{N}(v)^{s^{\prime} / 2} d \mu(\tau)-B(m, \mu) \Omega
$$

Again the assertion follows by unfolding the theta integral.

Remark 6.5. Let $\mu \in L^{\prime} / L$ and $m \in \partial_{F}^{-1}+Q(\mu)$ be totally positive. We have

$$
B(m, \mu)=-\frac{\operatorname{deg}(Z(m, \mu))}{\operatorname{vol}\left(X_{K}\right)}=-\frac{1}{2} A(m, \mu)
$$

Proof. Integrating the identity of (6.12) (respectively its analogue for $n \geq 1$ ) against $\Omega^{n-1}$ we obtain by means of (6.5) that

$$
\int_{X_{K}}\left(d d^{c} \Phi_{m, \mu}\right) \Omega^{n-1}=-B(m, \mu) \int_{X_{K}} \Omega^{n}
$$


On the other hand, Corollary 5.16 implies that the left hand side is equal to $\delta_{Z(m, \mu)}\left(\Omega^{n-1}\right)$. Consequently, $\operatorname{deg}(Z(m, \mu))=-B(m, \mu) \operatorname{vol}\left(X_{K}\right)$. (Alternatively, this can be proved as in [Ku3] Theorem 4.20, using (6.5) and the Thom form property of $\varphi_{K M .}$ )

6.4. Meromorphic modular forms and special divisors. We now use Theorem 6.4 to derive an analogue of Borcherds' result on automorphic products (see [Bo1], Theorem 13.3).

Lemma 6.6. Let $U \subset \mathbb{C}^{n}$ be a convex domain. Let $C$ be an analytic divisor on $U$, and let $\psi: U \backslash C \rightarrow \mathbb{R}$ be a $C^{2}$-function with a logarithmic singularity along $C$. If $\psi$ is pluriharmonic (i.e. $\partial \bar{\partial} \psi=0$ ), then there exists a meromorphic function $\Psi$ on $U$ such that $\psi=\log |\Psi|$.

Proof. By the assumption on $U$ we have $H^{1}\left(U, \mathcal{O}_{U}\right)=H^{2}(U, \mathbb{Z})=0$ and the multiplicative Cousin problem is universally solvable. Hence there is a meromorphic function $G$ on $U$ such that $C=\operatorname{div}(G)$. The assumption on $\psi$ implies that

$$
\psi-\log |G|
$$

extends to a pluriharmonic real analytic function on $U$. Since $U$ is simply connected, there exists a holomorphic function $H: U \rightarrow \mathbb{C}$ such that

$$
\Re(H)=\psi-\log |G|,
$$

see e.g. GR] Chapter IX, Section C. Rewriting this as

$$
\psi=\log \left|e^{H} \cdot G\right|,
$$

we see that we can take $\Psi=e^{H} \cdot G$.

The function $\Psi$ in the lemma has divisor $C$. By the maximum modulus principle, it is uniquely determined up to a constant of modulus 1 .

Lemma 6.7. (cp. Bo1 Lemma 13.1.) Let $r \in \mathbb{Q}$. Suppose that $\Psi$ is a meromorphic function on $\mathcal{H} \times H(\hat{\mathbb{Q}}) / K$ for which $|\Psi(z, h)| \cdot|y|^{r}$ is invariant under $H(\mathbb{Q})$. Then there exists a unitary multiplier system $\chi: H(\mathbb{Q}) \times H(\hat{\mathbb{Q}}) \rightarrow \mathbb{C}^{\times}$of weight $r$ such that $\Psi$ is a meromorphic modular form of weight $r$, level $K$, and multiplier system $\chi$.

Proof. The hypothesis implies that for every $\gamma \in H(\mathbb{Q})$ and $h \in H(\hat{\mathbb{Q}})$, the function

$$
\frac{\Psi(\gamma z, \gamma h)}{\Psi(z, h)} j(\gamma, z)^{-r}
$$

is holomorphic on $\mathbb{D}$ and has constant modulus 1 . By the maximum modulus principle it has to be constant, say equal to $\chi(\gamma, h)$. The right-invariance of $\Psi$ under $K$ implies that $\chi$ is right-invariant under $K$. Moreover, it is easily checked that $\chi$ satisfies the cocycle condition of a multiplier system.

Theorem 6.8. Let

$$
f=\sum_{\mu \in L^{\prime} / L} \sum_{m \gg 0} c(m, \mu) f_{m, \mu}(\tau) \in M_{k, \bar{\rho}_{L}}^{!}
$$

be a weakly holomorphic Whittaker form with coefficients $c(m, \mu) \in \mathbb{Z}$. Then there exists a function $\Psi(z, h, f)$ on $\mathcal{H} \times H(\hat{\mathbb{Q}})$ with the following properties: 
(i) $\Psi$ is a meromorphic modular form for $H(\mathbb{Q})$ of weight $-B(f)$ and level $K$ with a unitary multiplier system of finite order.

(ii) The divisor of $\Psi$ is equal to $Z(f)$.

(iii) The Petersson metric of $\Psi$ is given by

$$
-\log \|\Psi(z, h, f)\|_{P e t}^{2}=\Phi(z, h, f) .
$$

Proof. We use Theorem 6.4. The assumption that $f$ is weakly holomorphic means that $\xi_{k}(f)=0$. Consequently, we have

$$
\begin{aligned}
d d^{c} \Phi(z, h, f) & =-B(f) \Omega \\
& =B(f) d d^{c} \log |y|^{2} .
\end{aligned}
$$

Hence the function $\Phi(z, h, f)-B(f) \log |y|^{2}$ is pluriharmonic on $\mathcal{H}$ and has a logarithmic singularity along $-2 Z(f)$. According to Lemma 6.6 there exists a meromorphic function $\Psi(z, h, f)$ on $\mathcal{H} \times H(\hat{\mathbb{Q}}) / K$ such that

$$
\Phi(z, h, f)-B(f) \log |y|^{2}=-2 \log |\Psi(z, h, f)| .
$$

So $\Phi(z, h, f)=-\log \|\Psi(z, h, f)\|_{P e t}^{2}$, where the Petersson metric is in weight $-B(f)$. By construction, the divisor of $\Psi$ is equal to $Z(f)$.

The invariance properties of $\Phi(z, h, f)$ and Lemma 6.7 imply that there is a unitary multiplier system $\chi$ such that $\Psi$ is a meromorphic modular form of weight $-B(f)$, level $K$, and multiplier system $\chi$.

When $n>2$, the Lie group $H(\mathbb{R})$ has no almost simple factor of real rank 1 . Hence, according to Mar (Proposition 6.19 on p. 333), the multiplier system $\chi$ has finite order. When $n \leq 2$ we will prove that $\chi$ has finite order in the next section by means of the embedding trick, see Corollary 7.4 .

Remark 6.9. When $d=1$, then Theorem 6.8 is compatible (up to a constant) via Proposition 4.6 with the Borcherds lift of weakly holomorphic modular forms [Bo1, Theorem 13.3]. This follows from [Br], Proposition 2.11.

\section{Modularity of SPECIAL Divisors}

We first assume that $n>2$. We use Theorem 6.8 to prove that the generating series of special divisors is a Hilbert modular form of weight $\kappa$ with values in the Chow group. This result is proved in [YZZ] using the modularity result for the cohomology classes of Kudla-Millson [KM1, KM2, KM3]. Our proof is a variant of the proof that Borcherds gave for $F=\mathbb{Q}$, see [Bo2].

Next we consider the cases of small dimension $n=1,2$. Following [YZZ] and [Bo1], the modularity result can be extended to this case by means of the embedding trick. As an application we prove the finiteness of the multiplier system of the meromorphic modular form $\Psi(z, h, f)$ of Theorem 6.8 for $n=1,2$. 
7.1. The case $n>2$. For a Schwartz function $\varphi \in S_{L} \subset S(V(\hat{F}))$ we consider the special divisors $Z(m, \varphi)$. According to Remark [5.6, the divisors

$$
Z^{0}(m, \varphi):=Z(m, \varphi)-\frac{\operatorname{deg} Z(m, \varphi)}{\operatorname{vol}\left(X_{K}\right)} c_{1}\left(\mathcal{M}_{1}\right)
$$

have degree 0 . We define the generating functions

$$
\begin{aligned}
A(\tau, \varphi)=A_{V}(\tau, \varphi) & :=-c_{1}\left(\mathcal{M}_{1}\right)+\sum_{m \gg 0} Z(m, \varphi) q^{m}, \\
A^{0}(\tau, \varphi)=A_{V}^{0}(\tau, \varphi) & :=\sum_{m \gg 0} Z^{0}(m, \varphi) q^{m},
\end{aligned}
$$

which we view as formal power series with coefficients in $\mathrm{CH}^{1}\left(X_{K}\right)$. Here $q^{m}:=e(\operatorname{tr}(m \tau))$ and $c_{1}\left(\mathcal{M}_{1}\right)$ is the Chern class in $\mathrm{CH}^{1}\left(X_{K}\right)$ of $\mathcal{M}_{1}$ given by the divisor of a rational section. Moreover, we define the $\rho_{L}$-valued generating functions

$$
\begin{aligned}
A(\tau) & =\sum_{\mu \in L^{\prime} / L} A\left(\tau, \chi_{\mu}\right) \chi_{\mu}, \\
A^{0}(\tau) & =\sum_{\mu \in L^{\prime} / L} A^{0}\left(\tau, \chi_{\mu}\right) \chi_{\mu} .
\end{aligned}
$$

When the Eisenstein series $E_{L}(\tau, \kappa)$ is holomorphic, then Remark 6.5 implies that

$$
A^{0}(\tau)=A(\tau)+c_{1}\left(\mathcal{M}_{1}\right) E_{L}(\tau, \kappa) .
$$

Theorem 7.1. The generating series $A^{0}(\tau)$ belongs to $S_{\kappa, \rho_{L}} \otimes \mathrm{CH}^{1}\left(X_{K}\right)$.

For the proof of the theorem we need the following linear algebra lemma.

Lemma 7.2. Let $X, Y$ be vector spaces over a field $E$, and let $\beta: X \times Y \rightarrow E$ be a non-degenerate bilinear form. Let $X_{1} \subset X$ be a subspace and put

$$
\begin{aligned}
X_{1}^{\perp} & =\left\{y \in Y ; \beta(x, y)=0 \text { for all } x \in X_{1}\right\}, \\
X_{1}^{\perp \perp} & =\left\{x \in X ; \beta(x, y)=0 \text { for all } y \in X_{1}^{\perp}\right\} .
\end{aligned}
$$

If $X_{1}$ is finite dimensional, then $X_{1}^{\perp \perp}=X_{1}$.

Proof of Theorem 7.1. We write $P_{L}$ for the vector space of $\rho_{L}$-valued formal power series with vanishing constant term, that is, the vector space of formal power series of the form

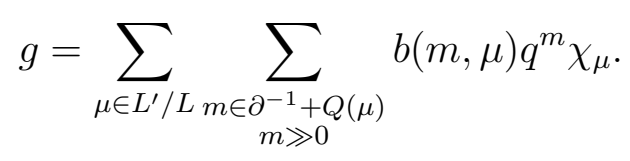

We may view $S_{\kappa, \rho_{L}}$ as a subspace of $P_{L}$ by taking the Fourier expansion of a cusp form. We extend the pairing $\{\cdot, \cdot\}$ between $H_{k, \bar{\rho}_{L}}$ and $S_{\kappa, \rho_{L}}$ defined in (4.22) to a non degenerate pairing between $H_{k, \rho_{L}}$ and $P_{L}$ using the formula (4.23). 
We apply Lemma 7.2 with $X=P_{L}, X_{1}=S_{\kappa, \rho_{L}}, Y=H_{k, \bar{\rho}_{L}}$, and the pairing $\{\cdot, \cdot\}$. We have that $M_{k, \bar{\rho}_{L}}^{!}=S_{\kappa, \rho_{L}}^{\perp}$. The generating series $A^{0}(\tau)$ is an element of $P_{L} \otimes \mathrm{CH}^{1}\left(X_{K}\right)$. According to the lemma it belongs to $S_{\kappa, \rho_{L}} \otimes \mathrm{CH}^{1}\left(X_{K}\right)$ if and only if

$$
\left\{A^{0}, f\right\}=0 \in \mathrm{CH}^{1}\left(X_{K}\right)_{\mathbb{C}}
$$

for all $f \in M_{k, \bar{\rho}_{L}}^{!}$. Adapting the argument of [McG], it can be proved that $S_{\kappa, \rho_{L}}$ has a basis of cusp forms with coefficients in $\mathbb{Z}$. Hence it suffices to verify (7.1) for those

$$
f=\sum_{\mu} \sum_{m \gg 0} c(m, \mu) f_{m, \mu}
$$

which are integral linear combinations of the $f_{m, \mu}$. So for such $f$ we have to show that

$$
\sum_{\mu} \sum_{m \gg 0} c(m, \mu) Z(m, \mu)=\sum_{\mu} \sum_{m \gg 0} c(m, \mu) \frac{\operatorname{deg} Z(m, \varphi)}{\operatorname{vol}\left(X_{K}\right)} c_{1}\left(\mathcal{M}_{1}\right) \in \mathrm{CH}^{1}\left(X_{K}\right) \mathbb{Q} .
$$

In view of Remark 6.5 this is equivalent to

$$
Z(f)=-B(f) c_{1}\left(\mathcal{M}_{1}\right) \in \mathrm{CH}^{1}\left(X_{K}\right)_{\mathbb{Q}} .
$$

But this relation is exactly produced by Theorem 6.8.

7.2. The embedding trick. Let $V_{1} \subset V$ be a quadratic subspace defined over $F$, and assume that $V_{1}$ has signature

$$
\left(\left(n_{1}, 2\right),\left(n_{1}+2,0\right), \ldots,\left(n_{1}+2,0\right)\right)
$$

with $1 \leq n_{1} \leq n$. Let $V_{2}=V_{1}^{\perp}$. Then $V_{2}$ is totally positive definite and $V=V_{1} \oplus V_{2}$. We view $H_{1}:=\operatorname{Res}_{F / \mathbb{Q}} \operatorname{GSpin}\left(V_{1}\right)$ as a subgroup of $H$ acting trivially on $V_{2}$ and put $K_{1}:=H_{1}(\hat{\mathbb{Q}}) \cap K$. We let $\mathbb{D}_{V_{1}}$ be the sub-Grassmannian of $\mathbb{D}$ given by the oriented negative definite 2-dimensional subspaces $z$ of $V_{1, \sigma_{1}} \subset V_{\sigma_{1}}$. We obtain an embedding of Shimura varieties

$$
\iota: X_{K_{1}, V_{1}}:=H_{1}(\mathbb{Q}) \backslash\left(\mathbb{D}_{V_{1}} \times H_{1}(\hat{\mathbb{Q}})\right) / K_{1} \longrightarrow X_{K, V} .
$$

It induces a pull-back homomorphism of the Chow groups

$$
\iota^{*}: \mathrm{CH}^{1}\left(X_{K, V}\right) \longrightarrow \mathrm{CH}^{1}\left(X_{K_{1}, V_{1}}\right) .
$$

The pull back of the generating series $A_{V}(\tau, \varphi)$ is computed in [YZZ, Proposition 3.1. Let $\varphi_{i} \in S\left(V_{i}(\hat{F})\right)$ and assume that $X_{K_{1}, V_{1}}$ is compact. Then

$$
\iota^{*}\left(A_{V}\left(\tau, \varphi_{1} \otimes \varphi_{2}\right)\right)=A_{V_{1}}\left(\tau, \varphi_{1}\right) \cdot \theta_{S, V_{2}}\left(\tau ; \varphi_{2}\right),
$$

where

$$
\theta_{S, V_{2}}\left(\tau ; \varphi_{2}\right)=\sum_{\lambda \in V_{2}(F)} \varphi_{2}(\lambda) e(\operatorname{tr} Q(\lambda) \tau)
$$

is the usual theta series of the positive definite quadratic space $V_{2}$. By embedding quadratic spaces over $F$ of dimension $n=1$ or 2 into larger spaces, employing the pull back-formula (7.2), and varying $\varphi_{2}$, one obtains (see [YZZ, proof of Theorem 1.3):

Proposition 7.3. Theorem 7.1 also holds for $n=1,2$. 
Corollary 7.4. Let $f \in M_{k, \bar{\rho}_{L}}^{!}$be a weakly holomorphic Whittaker form as in Theorem 6.8 and let $\Psi(z, h, f)$ be the corresponding meromorphic modular form for $H(\mathbb{Q})$. The multiplier system of $\Psi$ has also finite order when $n=1,2$.

Proof. Since $f$ is weakly holomorphic and $A^{0} \in S_{\kappa, \rho_{L}} \otimes \mathrm{CH}^{1}\left(X_{K}\right)$ by Proposition 7.3 , we have $\left\{A^{0}, f\right\}=0 \in \mathrm{CH}^{1}\left(X_{K}\right)_{\mathbb{Q}}$. This is equivalent to

$$
Z(f)=-B(f) c_{1}\left(\mathcal{M}_{1}\right) \in \mathrm{CH}^{1}\left(X_{K}\right)_{\mathbb{Q}} .
$$

So there is a meromorphic modular form $\tilde{\Psi}$ of weight $-B(f)$ and level $K$ with a multiplier system of finite order such that $\operatorname{div}(\tilde{\Psi})=Z(f)$. Consequently, $\Psi / \tilde{\Psi}$ is a holomorphic modular form of weight 0 with no zeros and a multiplier system of possibly infinite order. But it is easily seen that such a modular form must be constant. This proves the corollary.

\section{EXAMPLES}

Here we give some examples illustrating Theorem 6.8 and Theorem 7.1

8.1. Shimura curves. Let $B / F$ be a quaternion algebra which is split at $\sigma_{1}$ and ramified at all the other real places of $F$. Let $\delta \in \partial_{F}^{-1}$ be an element such that $\sigma_{i}(\delta)>0$ for $i=2, \ldots, d$. We write $\mathrm{N}(x)$ for the reduced norm of $x \in B$. Then $(B, \delta \mathrm{N})$ is a quadratic space over $F$ of signature $((2,2),(4,0), \ldots,(4,0))$. The group GSpin $(B)$ can be computed using similar arguments as in $\mathrm{KuRa} \S 0$. One finds that

$$
\operatorname{GSpin}(B) \cong\left\{\left(g_{1}, g_{2}\right) \in B^{\times} \times B^{\times} ; \mathrm{N}\left(g_{1}\right)=\mathrm{N}\left(g_{2}\right)\right\} .
$$

Under this identification the natural action of $\mathrm{GSpin}(B)$ on $B$ is identified with $\left(g_{1}, g_{2}\right) \cdot b=$ $g_{1} b g_{2}^{-1}$ for $b \in B$. We may view an $\mathcal{O}_{F}$-order $\mathcal{O} \subset B$ as an $\mathcal{O}_{F}$-lattice in the quadratic space $B$. The Shimura variety $X_{K}$ can be viewed as the product of two Shimura curves or as product of a Shimura curve with itself depending on the choice of the compact open subgroup $K$.

The subspace $B^{0} \subset B$ of trace zero elements of $B$ defines a quadratic subspace. When $\sigma_{1}(\delta)$ is also positive, it has signature $((1,2),(3,0), \ldots,(3,0))$. We may identify

$$
\operatorname{GSpin}\left(B^{0}\right) \cong B^{\times}
$$

the action on $B^{0}$ being given by conjugation. So Theorem 6.8 gives rise to automorphic forms on Shimura curves over $F$ with known divisor supported on special divisors.

It is interesting to consider the examples of Shimura curves investigated in [E] in that way. Here we briefly discuss the Shimura curve $X$ associated to the triangle group $G_{2,3,7}$, see [El] Section 5.3. It is a genus zero curve with a number of striking properties. For instance, the minimal quotient area of a discrete subgroup of $\mathrm{PSL}_{2}(\mathbb{R})$ is $1 / 42$, and it is only attained by the triangle group $G_{2,3,7}$. Let $F$ be the totally real cubic field $\mathbb{Q}(\cos (2 \pi / 7))=$ $\mathbb{Q}[x] /\left(x^{3}+x^{2}-2 x-1\right)$. It has discriminant 49 and class number 1 . The inverse different $\partial_{F}^{-1}$ has a generator $\delta$ such that $\sigma_{i}(\delta)>0$ for $i=2,3$. Let $B$ be the quaternion algebra over $F$ which is ramified at exactly the real places $\sigma_{2}, \sigma_{3}$. We view $(B, \delta \mathrm{N})$ as a quadratic space over $F$ as above. Let $L$ be a maximal $\mathcal{O}_{F}$-order of $B$. Then at all finite places $\mathfrak{p}$ of $F$ the lattice $L_{\mathfrak{p}}$ is isomorphic to the $2 \times 2$ matrices with entries in $\mathcal{O}_{F, \mathfrak{p}}$. This implies 
that $L$ is even unimodular and the corresponding Weil representation $\rho_{L}$ is trivial. The underlying lattice $\left(L, Q_{\mathbb{Q}}\right)$ over $\mathbb{Z}$ is isometric to $E_{8} \oplus H \oplus H$, where $H$ denotes a hyperbolic plane over $\mathbb{Z}$. We let $K \subset H(\hat{\mathbb{Q}})$ be the stabilizer of the lattice $\hat{L}$. Then the corresponding Shimura variety $X_{K}$ is isomorphic to $X \times X$, where $X$ is the quotient of $\mathbb{H}$ by the group of units of norm 1 of $L$.

The Jacquet-Langlands correspondence provides an isomorphism between the space of Hilbert cusp forms $S_{\kappa}$ of parallel weight $\kappa=(2,2,2)$ for $\mathrm{SL}_{2}\left(\mathcal{O}_{F}\right)$ and the space of holomorphic differential 1-forms on $X$. Since $X$ has genus 0 , we see that $S_{\kappa}=\{0\}$. Consequently, any harmonic Whittaker form $f$ of weight $k=(0,2,2)$ is weakly holomorphic. Its regularized theta lift gives rise to a meromorphic modular form of weight $-B(f)$ on $X_{K}$. Its divisor $Z(f)$ is a linear combination of Hecke correspondences. The generating series $A(\tau)$ of special divisors is equal to

$$
c_{1}\left(\mathcal{M}_{1}\right) E_{L}(\tau, \kappa)
$$

For any totally positive $m \in \partial_{F}^{-1}$ there exists a holomorphic modular form $\Psi_{m}$ on $X_{K}$ with divisor $Z(m, 0)$ and weight $B(m, 0)$, given by Theorem 6.8. These modular forms are analogues of the form $j\left(z_{1}\right)-j\left(z_{2}\right)$ and its multiplicative Hecke translates on $Y(1) \times Y(1)$, where $j$ denotes the classical $j$-function and $Y(1)=\mathrm{SL}_{2}(\mathbb{Z}) \backslash \mathbb{H}$. In view of the work of Gross and Zagier on singular moduli, it would be interesting to compute the CM values of the functions $\Psi_{m}$.

Similar unimodular lattices can be constructed over any totally real field of odd degree for which the different is a principal ideal in the narrow sense. But clearly the space of cusp forms for $\mathrm{SL}_{2}\left(\mathcal{O}_{F}\right)$ of parallel weight 2 will be non-trivial in general.

8.2. Even unimodular lattices over real quadratic fields. It would be interesting to have some existence or classification results for even unimodular $\mathcal{O}_{F}$-lattices (in the sense of Section 2.2), since the Weil representation $\rho_{L}$ is trivial in this case. Adapting the arguments of Scha] and [Ch], one can obtain some results in this direction. (Notice that the setup in these references is slightly different from ours. For instance, they consider $\mathcal{O}_{F}$-valued quadratic forms and define the dual lattice as the $\mathcal{O}_{F}$-dual.)

Here we briefly consider the case where $F$ is a real quadratic field of discriminant $D$.

Lemma 8.1. There exists an even unimodular $\mathcal{O}_{F}$-lattice of signature $((n, 2),(n+2,0))$ if and only if $n$ is divisible by 4 and $\mathcal{O}_{F}$ contains a totally positive unit $\varepsilon$ such that $-\varepsilon$ is a square modulo $4 \mathcal{O}_{F}$.

Proof. A lattice $(L, Q)$ is even unimodular of signature $((n, 2),(n+2,0))$ in the sense of Section 2.2 , if and only if $(L, \sqrt{D} Q)$ is a unimodular lattice of signature $((n, 2),(0, n+2))$ in the sense of [Ch]. Hence the lemma follows from Theorem 3 in [Ch].

Remark 8.2. i) If $F=\mathbb{Q}(\sqrt{a})$ with $a>0$ squarefree and $a \equiv 3(\bmod 4)$ then the condition of the lemma is always fulfilled when $n$ is divisible by 4 . In this case we can take $\varepsilon=1$.

ii) If the fundamental unit of $F$ has norm -1 , then the condition of the lemma is never fulfilled, since every totally positive unit is a square and therefore -1 would have to be a square modulo $4 \mathcal{O}_{F}$. This is not the case as an elementary computation shows. 
iii) If $\varepsilon \in \mathcal{O}_{F}$ is a totally positive unit such that $-\varepsilon=\alpha^{2}-4 \beta$ with $\alpha, \beta \in \mathcal{O}_{F}$, then the lattice $L_{1}$ given by the Gram matrix

$$
\frac{-1}{\sqrt{D}}\left(\begin{array}{cc}
2 & \alpha \\
\alpha & 2 \beta
\end{array}\right)
$$

is even unimodular of signature $((0,2),(2,0))$.

It also follows from Theorem 3 in $\mathrm{Ch}$ that for any real quadratic field $F$ there exists an even unimodular $\mathcal{O}_{F}$-lattice $L_{0}$ of signature $((4,0),(4,0))$. The corresponding lattice over $\mathbb{Z}$ is isometric to $E_{8}$. One can construct such a lattice explicitly by modifying the construction of Scha] Section 3. For instance, for $F=\mathbb{Q}(\sqrt{3})$, one can take the lattice with the Gram matrix

$$
\left(\begin{array}{cccc}
-\sqrt{3}+2 & \frac{\sqrt{3}-1}{2} & \frac{-\sqrt{3}+3}{6} & \frac{\sqrt{3}}{6} \\
\frac{\sqrt{3}-1}{2} & 3 & \frac{\sqrt{3}}{2} & \frac{-\sqrt{3}-3}{6} \\
\frac{-\sqrt{3}+3}{6} & \frac{\sqrt{3}}{2} & 1 & \frac{\sqrt{3}+1}{2} \\
\frac{\sqrt{3}}{6} & \frac{-\sqrt{3}-3}{6} & \frac{\sqrt{3}+1}{2} & 2+\sqrt{3}
\end{array}\right)
$$

If $n$ is divisible by 4 and $F$ satisfies the condition of the lemma, then $L=L_{0}^{\oplus n / 4} \oplus L_{1}$ is even unimodular of signature $((n, 2),(n+2,0))$.

Let now $F=\mathbb{Q}(\sqrt{3})$ and let $L$ be the lattice $L_{0} \oplus L_{1}$ as above. Let $K \subset H(\hat{\mathbb{Q}})$ be the stabilizer of $\hat{L}$. One can show that the space of Hilbert cusp forms $S_{\kappa}$ of parallel weight $\kappa=(3,3)$ for $\mathrm{SL}_{2}\left(\mathcal{O}_{F}\right)$ vanishes. Consequently, any harmonic Whittaker form $f$ of weight $k=(-1,3)$ is weakly holomorphic. For any totally positive $m \in \partial_{F}^{-1}$ there exists a holomorphic modular form $\Psi_{m}$ on $X_{K}$ with divisor $Z(m, 0)$ and weight $B(m, 0)$, given by Theorem 6.8. The generating series $A(\tau)$ of special divisors is equal to $c_{1}\left(\mathcal{M}_{1}\right) E_{L}(\tau, \kappa)$.

\section{REFERENCES}

[AbSt] M. Abramowitz and I. Stegun, Pocketbook of Mathematical Functions, Verlag Harri Deutsch, Thun (1984).

[AF] D. Allcock and E. Freitag, Cubic Surfaces and Borcherds Products, Commentarii Math. Helv. 77 (2002), 270-296.

[Bo1] R. Borcherds, Automorphic forms with singularities on Grassmannians, Inv. Math. 132 (1998), 491-562.

[Bo2] R. E. Borcherds, The Gross-Kohnen-Zagier theorem in higher dimensions, Duke Math. J. 97 (1999), 219-233.

[Bo3] R. E. Borcherds, Automorphic forms and Lie algebras, Current Developments in mathematics 1996, International Press (1998).

[Br] J. H. Bruinier, Borcherds products on $\mathrm{O}(2, l)$ and Chern classes of Heegner divisors, Springer Lecture Notes in Mathematics 1780, Springer-Verlag (2002).

[BBK] J. H. Bruinier, J. Burgos, and U. Kühn, Borcherds products and arithmetic intersection theory on Hilbert modular surfaces, Duke Math. J. 139 (2007), 1-88.

[BF] J. H. Bruinier and J. Funke, On two geometric theta lifts, Duke Math. Journal. 125 (2004), 45-90.

[BK] J. H. Bruinier and U. Kühn, Integrals of automorphic Green's functions associated to Heegner divisors, Int. Math. Res. Not. 2003:31 (2003), 1687-1729. 
[BY1] J. H. Bruinier and T. Yang, Faltings heights of CM cycles and derivatives of $L$-functions, Invent. Math. 177 (2009), 631-681.

[BY2] J. H. Bruinier and T. Yang, CM values of automorphic Green functions on orthogonal groups over totally real fields, arXiv:1004.3720, preprint (2010).

[BKK] J. Burgos, J. Kramer, and U. Kühn, Cohomological arithmetic Chow groups, J. Inst. Math. Jussieu. 6, 1-178 (2007).

[Ch] K.-S. Chang, Diskriminanten und Signaturen gerader quadratischer Formen, Arch. Math. (Basel) 21 (1970), 59-65.

[El] N. D. Elkies, Shimura Curve Computations. In Algorithmic number theory (Portland, OR, 1998), 1-47, Lecture Notes in Comput. Sci. 1423, Springer, Berlin (1998).

[Er1] A. Erdélyi, W. Magnus, F. Oberhettinger and F. G. Tricomi, Higher Transcendental Functions, McGraw-Hill (1953).

[Er2] A. Erdélyi, W. Magnus, F. Oberhettinger and F. G. Tricomi, Tables of Integral Transforms, vol. I, McGraw-Hill (1954).

[Fr] E. Freitag, Hilbert Modular Forms, Springer-Verlag, Berlin (1990).

[Ga] P. B. Garrett, Holomorphic Hilbert Modular Forms, Brooks/Cole Publishing Company (1990).

[Go] E. Goren, Lectures on Hilbert modular varieties and modular forms, CRM monograph series 14 (2001).

[GN] V. Gritsenko and V. Nikulin, Automorphic forms and Lorentzian Kac-Moody algebras. Part II, Intern. J. of Math. 9 (1998), 201-275.

[GKZ] B. Gross, W. Kohnen, and D. Zagier, Heegner points and derivatives of $L$-series. II. Math. Ann. 278 (1987), 497-562.

[GZ] B. Gross and D. Zagier, Heegner points and derivatives of $L$-series, Invent. Math. 84 (1986), $225-320$.

[GR] R. C. Gunning and H. Rossi, Analytic functions of several complex variables, Prentice-Hall (1965).

[HM] J. Harvey and G. Moore, Algebras, BPS states, and strings, Nuclear Phys. B 463 (1996), no. 2-3, 315-368.

[He] D. A. Hejhal, The Selberg Trace Formula for PSL $(2, \mathbb{R})$, Lecture Notes in Mathematics 1001, Springer-Verlag (1983).

[Ku1] S. Kudla, Algebraic cycles on Shimura varieties of orthogonal type. Duke Math. J. 86 (1997), no. $1,39-78$.

[Ku2] S. Kudla, Central derivatives of Eisenstein series and height pairings. Ann. of Math. (2) 146 (1997), 545-646.

[Ku3] S. Kudla, Integrals of Borcherds forms, Compositio Math. 137 (2003), 293-349.

[Ku4] S. Kudla, Special cycles and derivatives of Eisenstein series, in Heegner points and Rankin Lseries, Math. Sci. Res. Inst. Publ. 49, Cambridge University Press, Cambridge (2004).

[KM1] S. Kudla and J. Millson, The theta correspondence and harmonic forms I, Math. Ann. 274, (1986), 353-378.

[KM2] S. Kudla and J. Millson, The theta correspondence and harmonic forms II, Math. Ann. 277, (1987), 267-314.

[KM3] S. Kudla and J. Millson, Intersection numbers of cycles on locally symmetric spaces and Fourier coefficients of holomorphic modular forms in several complex variables, IHES Publi. Math. $\mathbf{7 1}$ (1990), 121-172.

[KR] S. Kudla and S. Rallis, On the Weil-Siegel formula, J. Reine Angew. Math. 387 (1988), 1-68.

[KuRa] S. Kudla and M. Rapoport, Arithmetic Hirzebruch-Zagier divisors, J. Reine Angew. Math. 515 (1999), 155-244.

[KRY] S. Kudla, M. Rapoport, and T.H. Yang, Modular forms and special cycles on Shimura curves, Annals of Math. Studies series, vol 161, Princeton Univ. Publ., 2006.

[KY] S. Kudla and T. Yang, Derivatives of Eisenstein series, in preparation. 
[Luo] W. Luo, Poincaré series and Hilbert modular forms, Ramanujan J. 7 (2003), 129-140.

[Mar] G. A. Margulis, Discrete Subgroups of Semisimple Lie Groups, Springer-Verlag (1991).

[McG] W. J. McGraw, The rationality of vector valued modular forms associated with the Weil representation, Math. Ann. 326 (2003), 105-122.

[MW] R. Miatello and N. R. Wallach, The resolvent of the Laplacian on locally symmetric spaces, J. Differential Geom. 36 (1992), 663-698.

[OT] T. Oda and M. Tsuzuki, Automorphic Green functions associated with the secondary spherical functions. Publ. Res. Inst. Math. Sci. 39 (2003), 451-533.

[Scha] R. Scharlau, Unimodular lattices over real quadratic fields, Math. Z. 216 (1994), 437-452.

[Scho] J. Schofer, Borcherds forms and generalizations of singular moduli, J. Reine Angew. Math. 629 (2009), 1-36.

[Sh] G. Shimura, Introduction to the Arithmetic Theory of Automorphic Functions, Princeton University Press, Princeton (1971).

[Shu] M. A. Shubin, Pseudodifferential Operators and Spectral Theory, Springer-Verlag (2001).

[SABK] C. Soulé, D. Abramovich, J.-F. Burnol, and J. Kramer, Lectures on Arakelov Geometry, Cambridge Studies in Advanced Mathematics 33, Cambridge University Press, Cambridge (1992).

[We1] A. Weil, Sur certaines groupes d'operateurs unitaires, Acta Math. 111 (1965) 143-211.

[We2] A. Weil, Sur la formule de Siegel dans la théorie des groupes classiques, Acta Math. 113 (1965) $1-87$.

[YZZ] X. Yuan, W. Zhang and S. Zhang, The Gross-Kohnen-Zagier Theorem over Totally Real Fields, Compositio Math. 145 (2009), 11471162.

Fachbereich Mathematik, Technische Universität Darmstadt, Schlossgartenstrasse 7, D-64289 Darmstadt, Germany

E-mail address: bruinier@mathematik.tu-darmstadt.de 